\title{
Ethical Approaches to Gathering Information from Children and Adolescents in International Settings: Guidelines and Resources
}

Katie D. Schenk

Population Council

Jan Williamson

Follow this and additional works at: https://knowledgecommons.popcouncil.org/departments_sbsr-hiv

Part of the Demography, Population, and Ecology Commons, Family, Life Course, and Society Commons, International Public Health Commons, and the Medicine and Health Commons How does access to this work benefit you? Let us know!

\section{Recommended Citation}

Schenk, Katie D. and Jan Williamson. 2005. "Ethical Approaches to Gathering Information from Children and Adolescents in International Settings: Guidelines and Resources." Washington, DC: Population Council. 


\section{Ethical Approaches to \\ Gathering Information from \\ Children and Adolescents in \\ International Settings:}

Guidelines and Resources

\section{Horizons \\ Population Council \\ IMPACT}

Family Health International

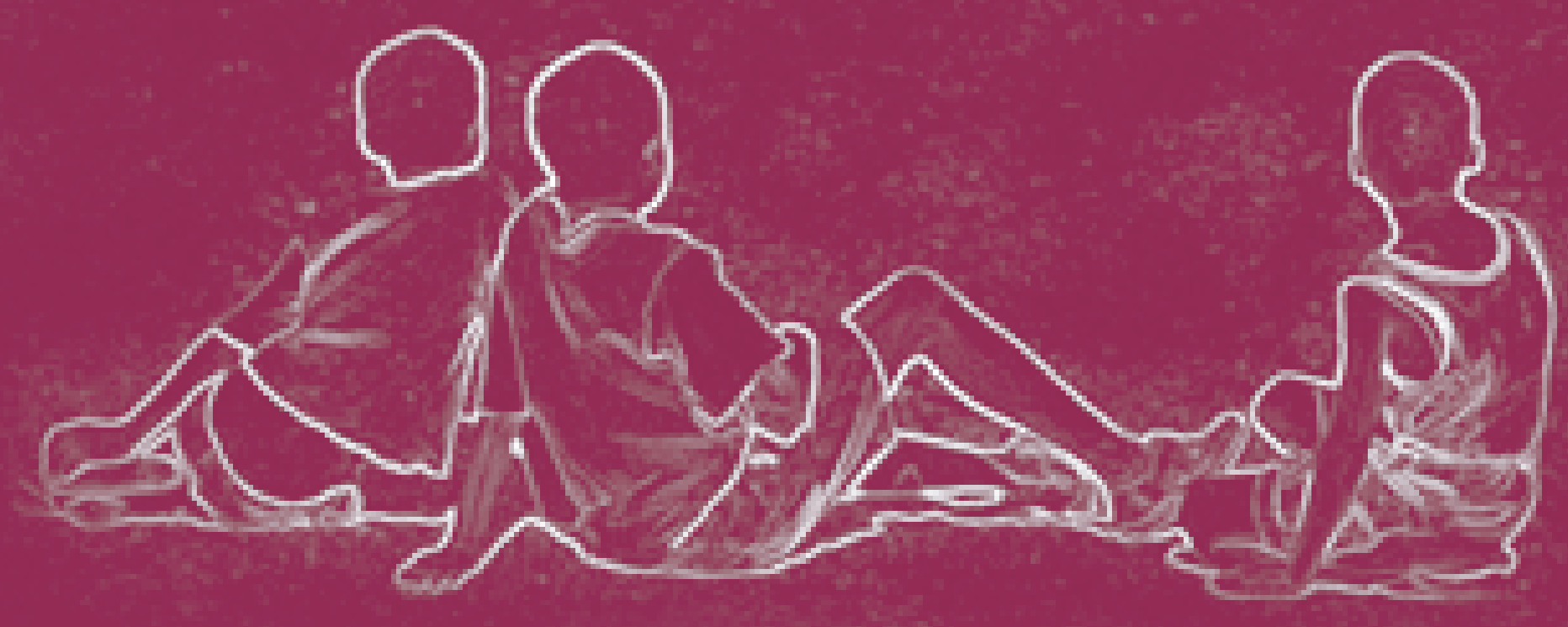





\title{
Ethical Approaches to Gathering I nformation from Children and Adolescents in I nternational Settings:
}

\section{Guidelines and Resources}

\author{
Katie Schenk \\ Jan Williamson
}

\section{Horizons}
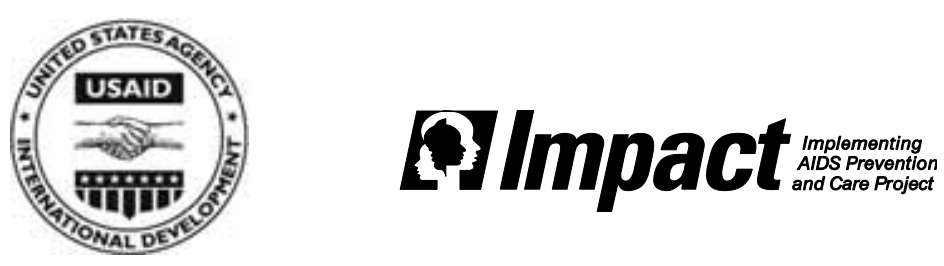

(2) Population Council
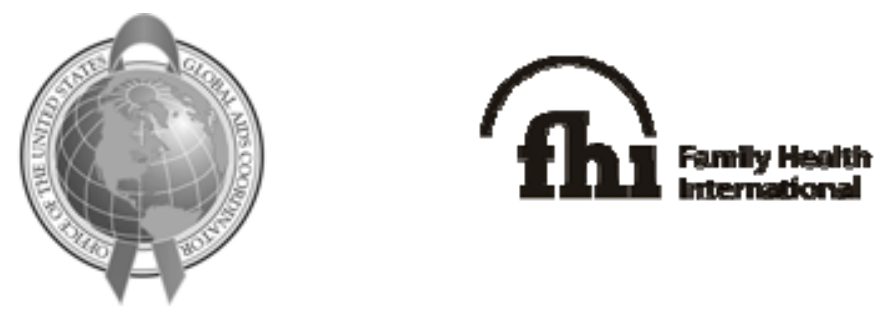
ISBN 0-87834-113-7

Suggested citation: Schenk, Katie and Jan Williamson. 2005. Ethical A pproaches to Gathering Information from Children and A dolescents in International Settings: Guidelines and Resources. Washington, DC: Population Council.

The development of this publication was supported by the Horizons Program and IMPACT. Horizons is implemented by the Population Council in collaboration with the International Center for Research on Women, International HIV/ AIDS Alliance, Program for A ppropriate Technology in Health, Tulane University, Family Health International, and Johns Hopkins University. Horizons is funded by the President's Emergency Plan for AIDS Relief through the U.S. Agency for International Development, under the terms of HRN-A-00-97-00012-00.

IMPACT is implemented by Family Heal th International in collaboration with Management Sciences for Health, Population Services International, Program for A ppropriate Technology in Health, University of North Carolina at Chapel Hill, and the Institute for Tropical Medicine. IMPACT is funded by the President's Emergency Plan for AIDS Relief through the U.S. Agency for International Development, under the terms of HRN-A-00-97-00017-00.

The opinions expressed herein are those of the authors and do not necessarily reflect the views of the U.S. Agency for International Development.

PDF version published in August 2005.

Cover design by Sherry Hutchinson.

Original photograph by KatieSchenk.

Copyright @ 2005 The Population Council Inc. All rights reserved.

This material may be quoted or reproduced without charge for noncommercial purposes only; however, please acknowledge source and send a copy of reproduced material to Horizons.

Communications Unit/ Horizons

4301 Connecticut A ve NW, Suite 280

Washington, DC 20008

USA

horizons@pcdc.org

http:// www.popcouncil.org/ horizons 


\section{Acknowledgments}

This document was written by Katie Schenk (Horizons/ Population Council) and Jan Williamson (consultant) under the guidance of the Steering Group. The members of the Steering Group and their affiliations at the time of their involvement were Johannes van Dam (Horizons/ Population Council), Linda Sussman (USAID), Gina Dallabetta and Suzi Peel (IMPACT/ FHI), and Roeland Monasch (UNICEF).

Initial ideas for this publication were discussed at an Expert Consultation meeting on gathering information from children in N ovember 2003 attended by Steering Group members and Isolde Birdthistle (London School of Hygiene and Tropical Medicine); Nicollette Borek (NIDA/ NIH); Minki Chatterji (Community REACH Project); Katherine Davenny (NIH); Joan Duncan (Long Island University); Kate Harrison (International HIV/ AIDS Alliance); KyleJemison (Catholic Relief Services/ STRIVE); Millicent Obaso (A HEAD Inc.); Chloe O'Gara (AED); Leslie Snider (Tulane University); Diane Swales (Save the Children); Mike Wessells (Randolph Macon University/ Christian Children's Fund); and David Borasky, Inoussa Kabore, Nicole Kouassi, Robert Magnani, Susan Mathew, Susan Pobywajlo, Dimitri Prybylski, Julie Victor-A huchogu, and Joshua Volle (FHI).

Case studies for this publication were contributed by the individuals listed above and Angela Bannerman (Planned Parenthood Association of Ghana); Jonathan Brakarsh and Jennifer Lentfer (Catholic Relief Services/ STRIVE); David Tolfree and Gillian Mann (Save the Children Sweden); Anne Fiedler (Straight Talk Uganda); Tracy Dolan (Christian Children's Fund); Karen Manda, Francesca Stuer, and Nancy Williamson (FHI); Pirkko Jokinen (Savonia Polytechnic); Susan Adamchak (formerly of Frontiers/ FHI); Tricia Smith (REPSSI); Heather Montgomery (Open University); Carol Culler (USAID/ M ozambique); Laelia Gilborn (formerly of Horizons/ Population Council); Samson Bwalya Chama (Virginia Commonwealth University), and Sharry Erzinger (University of Colorado at Denver). The quotes from children and adolescents that appear in this document come from UNICEF's Voices of Youth website (http:/ / www.unicef.org/ voy/ ).

Thanks also to Judith Bruce, Barbara Friedland, Heidi Jones, Bob Miller, Nancy Sloan, and John Townsend (Population Council); Gretchen Bachman and Sara Bowsky (FHI); Edward Marienga (Family Planning Association of Kenya); and Ellen Weiss (Horizons/ International Center for Research on Women) for helpful comments.

The authors would particularly like to express gratitude to UNICEF for their support throughout this process. UNICEF involvement was coordinated by Roeland Monasch. Thanks to Kate Alley, Trevor Croft, Edilberto Loaiza, Mary Mahy, Peter Mason, Geetanjali Narayan, Elizabeth Santucci, Miriam Temin, and A lexandra Yuster for their constructive reviews.

Many thanks to Jessica Clarke, Sherry Hutchinson, Sharon Lamberton, Alison Lee, and Barbara Minich for editorial, design, and administrative support. 



\section{Table of Contents}

\section{Acknowledgments}

Working Definitions $\quad$ i

Abbreviations and Acronyms iii

$\begin{array}{ll}\text { Preface } & \text { iv }\end{array}$

Using this Publication vi

Summary viii

$\begin{array}{ll}\text { Introduction } & 1\end{array}$

Part 1 - Key Considerations $\quad 3$

1. Basic ethical principles 3

2. Children's participation 5

3. Legal and professional requirements (including ethical supervision) 7

4. Cultureand gender considerations 11

5. Considerations for especially vulnerable children 12

Part 2 - Ethical Questions and Practical Guidelines 15

A. Is this activity necessary and justified? $\quad 15$

B. Is this activity designed to get valid information? 18

C. Have community and stakeholder concerns been considered? 23

D. Have adverse consequences of the activity been anticipated? 27

E. Are procedures in place to ensure that the activity proceeds ethically? 41

F. Is there a clear plan and adequate funding for follow-up activities? 53

$\begin{array}{ll}\text { Part } 3 \text { - Summary and Recommendations } & 57\end{array}$

$\begin{array}{ll}\text { Annexes } & 63\end{array}$

Annex 1: Child- and adolescent-focused resources 64

Annex 2: Legal and professional resources 66

Annex 3: Additional resources 70

Annex 4: Sample documents $\quad 72$ 



\section{Working Definitions}

People in different professional, geographical, or cultural groups are often confused by each other's use of language. Some groups use different words to mean the same thing. Others use the same word but interpret it in different ways.

Since this publication is aimed at people working with children and adolescents in a variety of settings, sensitivity and clear communication are required. This section explains technical words and related terms used within this document.

A buse: anything that individuals or institutions do or fail to do that directly or indirectly harms children or damages their prospects for safe and healthy development

A dditional consent: consent required from adults working with children, such as teachers, clergy, youth workers, and others, to gain access to gather information from children (see Guideline E1)

A dolescent: individual in the state of development between the onset of puberty and maturity; definitions vary according to culture and custom (WHO definition is from age 10 through age 19)

A ge of consent: age at which an individual may give consent to sexual activity with another person

A ge of majority: age at which an individual becomes a legal adult and gains full legal rights, such as the right to vote

A nonymity: conditions under which the identity of the participant is not collected and cannot be traced from the information provided (see Guideline D3)

A ssent: affirmative agreement of a child (see Guideline E1)

Child advocate: independent adult appointed to represent the best interests of a child who cannot legally give consent (see Guidel ine E4)

Child or minor: individual younger than age 18; definitions vary according to culture and custom

Community advisory board: committee of local community members and stakeholders responsible for overseeing the design and conduct of activities to protect participants from harm and ensuring that activities are culturally acceptable locally and in accordance with ethical standards; also known as local advisory board (see Part 1, Section 4, and Guideline C3)

Confidentiality: conditions under which the information reveal ed by an individual participant in a relationship of trust will not be disclosed to others without permission (see Guidelines D3 and D7)

Consent: affirmative agreement of an individual who has reached the legal age of majority (see GuidelineE1)

D issent: disagreement; withholding of consent (see Guideline E1)

D o no harm: basic ethical principle stating that the primary concern must al ways be to protect the participant from harm (see Part 1, Section 1)

Ethical standards: principles used to resolve questions that invoke responsibilities for the welfare of others or conflicts among loyalties to different groups 
Ethics: study of fundamental principles that define values and determine moral duties and obligations

Ethical review board: group of experienced professional experts overseeing the design and conduct of activities to protect participants from harm and ensure that legal and ethical standards are upheld; may also be known as an ethics committee (see Part 1, Section 3)

Exploitation: taking advantage of someone for one's own benefit

Foster parents: adults who care for a child who is not biological ly their own, acting in place of the child's parents through a formal or informal agreement

G atekeeper: individual with the authority to grant formal or informal permission or access to approach other individuals (see Guideline C1)

G uardian: individual authorized to give consent for participation on behalf of a child

Informed consent: process to ensure that each participating individual does so willingly and with adequate understanding (see GuidelineE1)

In loco parentis: Latin term meaning "in place of the parents" generally used to refer to a person, institution, or organization, such as an NGO or orphanage, that assumes the legal rights and responsibilities for children when parents are unable to do so

Minor: see "child"

Monitoring: regular oversight and measurement of the progress of an activity

N eglect: failure to provide for a child's basic needs, including physical, medical, educational, and emotional needs

Orphan: child whose mother or father (or both) has died

Parent: child's biological or adoptive parent

Participant: individual who provides data in the information-gathering activity; participants may include children and parents or guardians

Participation: taking part in decision-making and action

Permission: agreement of parent(s) or guardian(s) to participation by the child (see GuidelineE1)

Protocol: document that describes the objective, design, methodology, and management of an information-gathering activity used in programs and research

Psychosocial needs: needs relating to psychological and social wellbeing

Separated child: child who may have one or both parents living but who is in the care of another adult or an institution

Stakeholder: person or organization with an interest in an activity and affected by its outcome

Unaccompanied child: child who is not being cared for by any adult

Vulnerable child: child whose survival, wellbeing, or development is threatened; a term often used to refer to children affected by HIV and AIDS

Youth: a young person; definitions may vary according to culture and custom (WHO definition is from age 15 through age 24) 


\section{Abbreviations and Acronyms}

$\begin{array}{ll}\text { AIDS } & \text { Acquired immune deficiency syndrome } \\ \text { FHI } & \text { Family Health International } \\ \text { HIV } & \text { Human immunodeficiency virus } \\ \text { NGO } & \text { Nongovernmental organization } \\ \text { UN } & \text { United Nations } \\ \text { UNICEF } & \text { United Nations Children's Fund } \\ \text { USAID } & \text { United States Agency for International Development } \\ \text { WHO } & \text { World Health Organization }\end{array}$




\section{Preface}

To improve the lives of children and adolescents it is necessary to find out about their circumstances, their needs, and the services they require to meet their needs. Program managers and researchers who gather this information in order to develop and evaluate appropriate responses to the needs of children and adolescents must maintain high ethical standards in order to protect and respect them.

Observing ethical standards is important for all information gathering that involves people, including program monitoring and evaluation and research studies. However, extra precautions are needed to protect young people, who are especially vulnerable to exploitation, abuse, and other harmful outcomes.

Children and adolescents may suffer harm because they have less power and access to resources than adults. Thus, strategies used to gather information among adults cannot typically be used among children and adolescents without additional safeguards. This publication draws attention to commonly accepted principles of medical ethics and human rights as applied to the special needs of young people, and suggests measures that an adult working with children and adolescents must implement to prevent unintentional harm. Although careful planning is an important part of ethical practice, attention to these standards applies throughout the activity's duration.

Working ethically with children and adolescents to recognize and respond to their needs also requires program managers and researchers to seek out the views of children and adolescents when they are gathering information. This publication is intended to address a wide range of information-gathering activities that recognize and include the views of children and adolescents.

This publication was developed in response to a growing need for practical guidance on collecting information from and about young people. The initial need for such recommendations was identified in the context of activities being conducted among children and adolescents affected by HIV and AIDS. However, they apply equally well to gathering information from young people to address other health and social welfare conditions and difficult circumstances, such as those who have experienced abuse, trafficking, or displacement.

This publication is the result of an international consultative process among experts in different fields, including psychology, child development, sociology, anthropology, ethics, pediatric medicine, HIV/ AIDS programs, and social work. The process began with an Expert Consultation meeting, which took place in November 2003. Participants indicated that they valued this opportunity to discuss their work with colleagues in different fields and to share and learn from each other's experiences. Ethical A pproaches to Gathering Information from Children and A dolescents in International Settings: Guidelines and Resources is thus an initial step in the development of a new and rapidly evolving area of 
work focused on gathering information from children and adolescents, which is gaining increased attention.

This document aims to provide guidance through a maze of ethical issues that require consideration when working with young people. It is not a methodological handbook describing approaches to working with young people. Although methodological and ethical concerns may often overlap, for example when considering interview techniques suitable for use with children, investigators should refer to other sources for specific methodological guidance (see Annex 1 for additional resources).

Ethical practice often requires finding a balance among several different sets of demands. There are no easy answers to any of the questions discussed. The issues are presented in order to explore possible responses and promote discussion and collaboration among people who address them from different perspectives. Feedback on the questions raised in this document is welcome. Please send comments and suggestions to horizons@pcdc.org. 


\section{Using this Publication}

\section{Aims}

This publication aims to:

- Provide practical guidance to help program managers and researchers understand and uphold ethical standards when planning and implementing informationgathering activities among children and adolescents.

- Promote discussion of ethical issues when working with children and adolescents among people of different backgrounds and training who gather information for different purposes.

\section{Audience}

This publication is intended to be helpful to anyone who plans, manages, implements, analyzes, or funds any program or research activity that requires gathering information from children and adolescents, including monitoring and evaluation. The terms used here include:

- "Information gathering": describes any contact made by adults with children and adolescents for the purpose of collecting program or research data, including interviews, observations, and focus group discussions.

- "Investigator": refers to any staff member involved in the information-gathering activity, such as program managers and implementers, interviewers, focus group facilitators, clinicians, donors, researchers, and data analysts.

- "Children," "adolescents," and "youth": describe the young participants, informants, and respondents of information-gathering activities. See the working definitions on page i for more information.

\section{Structure}

Part 1 of this document presents some key issues that must be considered from the earliest stages of planning and throughout the information-gathering activity. These cross-cutting themes underlie the practical guidance that follows.

Part 2 introduces practical guidance for observing high ethical standards at specific stages of an information-gathering activity involving children and adolescents. These guidelines are presented using a question-and-answer format.

Part 3 summarizes the main recommendations and suggests roles for various staff members involved in activities gathering information from children and adolescents.

Key information from Parts 1 and 2 are highlighted in the summary on pages viii and ix. 


\section{Key to symbols}

"Lessons from experience" are indicated by this symbol. These case studies illustrate real-life struggles with ethical challenges. They are not examples of best practices: some frankly identify missed opportunities or oversights and trace the lessons that they learned.

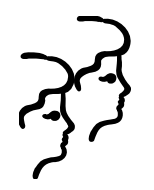

Opinion pieces, called "viewpoints," are indicated by this symbol. They offer the perspectives of children, adolescents, and adults who have considered ethical questions.

Sometimes considerations in this publication overlap, and material in a different section will be relevant to the question being discussed. These cross-references are indicated by this symbol in the text.

This publication does not provide general training on research ethics, participatory methods, or conducting community assessments. This symbol indicates where references to additional resources for these other purposes are provided in the Annexes. 


\section{Summary}

A. Make sure the information-gathering activity is necessary and justified.

- Before starting the activity, clearly define its intended purpose and audience, and make sure that there is sufficient staff and money to conduct it in an ethical manner.

* Only use direct methods with children if the required information is not otherwise available.

* If the information-gathering activity will not directly benefit the children and adolescents involved or their community, do not proceed.

B. Design the activity to get valid information.

- Develop a protocol to clarify aims and procedures for collecting, analyzing, and using the information to which all partners agree.

- A pply community definitions to set clear criteria for inclusion. Use existing records when possible, and recognize social and cultural barriers to participation. For surveys, use the minimum number of respondents to achieve demonstrable results.

* All tools, such as questionnaires, should be developed through discussions with experts. These tools should then be translated locally, back-translated, and field-tested.

* The use of a comparison group totally deprived of services is inappropriate with vulnerable children. A Iternative approaches should be explored to strengthen research findings. Comparison groups should be used only under careful ethical supervision.

C. Consult with community groups.

* Consult locally to determine who must give permission for the activity to proceed.

* Interviewers must be sensitive that they may be highly visible and a source of local interest. Clarify roles and expectations through community meetings and honor commitments.

* An independent local community stakeholder group should monitor activities.

D. Anticipate adverse consequences.

* In partnership with the community, anticipate all possible consequences for the children and adolescents involved. Do not proceed unless appropriate responses to potentially harmful consequences can be provided.

- Avoid stigma by holding community sensitization meetings and using community terminology.

* If the safety and security of children and adolescents cannot be assured, do not proceed.

* Interviewers should have experience working with children. They should be trained to respond to children's needs, and require ongoing supervision and support. If appropriately skilled interviewers are unavailable, do not proceed.

- In partnership with the community, determine what kind of follow-up is appropriate to respond to children's needs, recognizing age, gender, ethnicity, and so on. If appropriate support cannot be assured to meet the children's needs, do not proceed.

- Prepare a reaction plan to anticipate serious needs. If support for the child cannot be assured, do not proceed. 
* Confidentiality should be breached to provide immediate protection to the child or adolescent. Staff should make sure that participants are aware of this before asking for any information.

E. Conduct consent and interviewing procedures with sensitivity to children's specific needs.

* Children must give their agreement to participate, but consent is required from appropriate adults.

* Interviewers should make sure that children know they can stop or withdraw at any time.

* Investigators must provide children and adolescents and their parent or guardian with information about the activity in a manner appropriate to their culture and education. Consent forms and informational tools should be developed with community members and field-tested.

- Usean independent advocate to represent the views of children if there is any doubt about the protection provided by their guardian.

* Avoid efforts to unduly influence participation by the use of incentives. If incentives are used, they should be in line with local living standards.

- Interview procedures should reflect the need to protect the children and adolescents' best interests. Consult with community members to determine appropriate practices.

F. Confirm that all stakeholders understand the limits to the activity and next steps.

* Use appropriate procedures to maintain the safety and security of participants.

* Share findings with community members in an accessible, appropriate format.

\section{Key take-home messages}

1. Consider the following cross-cutting themes while planning and implementing the information-gathering activity: basic ethical principles; children's participation; legal and professional requirements, including ethical supervision; culture and gender; and especially vulnerable children.

2. Balance the need to maximize children's participation by hearing their own opinions on the issues affecting their lives with the need to minimize their exposure to harm.

3. Careful advance planning is crucial. Investigators are responsible for thinking through all possible consequences, both intentional and unintentional, of the information-gathering activity and for anticipating the effect of the activity on young people and their families. If appropriate safeguards cannot be put into place, the activity should not proceed.

4. D iscussions should be held with local community members, including children and adolescents, whenever possible. Community meetings at different stages of the information-gathering activity can serve a variety of purposes, including sensitization, review, and interpretation. These discussions can serve the dual purpose of improving adherence to ethical standards and improving the quality of the information gathered.

5. If there is any question about whether the data collection could be harmful to children, only begin the activity if services are in place to address possible consequences. If the information-gathering activity is not associated with a service, prepare referral information for children to reach the required support. Do not conduct the activity without functional support systems in place. 
x - Ethical Approaches to Gathering Information from Children and Adolescents in International Settings 


\section{Introduction}

- An interviewer is making household visits to monitor a children's nutrition program. She has explained to participants that the information she gathers during the interview is confidential. D uring one interview, she discovers that a child is being abused. W hat should she do? What are her responsibilities? With whom should this information be shared?

- A researcher is investigating literacy among children in an isolated village. She soon discovers that just asking the question, "D o you attend school?" causes some of the children to cry. They experience such extreme levels of poverty that they find it upsetting to even think about the school they cannot attend. H ow should the interviewer proceed?
Children and adolescents around the world face challenges in all aspects of their lives, including their health, education, and environment. These difficulties have often been made worse by the growing impact of HIV and AIDS, making them vulnerable to many economic and social pressures. People who develop and provide services to assist these young people must seek out their views to gather accurate and culturally specific information that will help them provide appropriate support.

Many difficult ethical dilemmas may arise when gathering this information among children and adolescents. Despite experience, education, and good intentions, skilled professionals often find themselves questioning how to proceed with their activities with young people. For example, without meaning to, interviewers can create anxiety or trigger grief even when they are trying to bring benefits to the lives of the young people. At the same time, recognizing children's opinions about the factors shaping their lives can bring surprising outcomes, making it important that they participate in designing activities.

This publication applies to a wide variety of information-gathering purposes, methods, and sources (see Box 1). It is intended to help in planning an information-gathering activity from its earliest stages through its implementation. These guidel ines remain relevant after completion of the activity to the release of the findings and beyond.

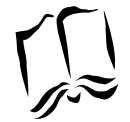

See Annex 1.1 for more information about issues affecting children and approaches to working with children 
Box 1 Information-gathering purposes, methods, and sources: Some examples

\begin{tabular}{|c|c|c|}
\hline $\begin{array}{l}\text { Information gathering may } \\
\text { involve a variety of } \\
\text { purposes... }\end{array}$ & ...using a variety of methods... & ...from a variety of sources. \\
\hline $\begin{array}{l}\text { - to identify and assess needs } \\
\text { - to develop and implement } \\
\text { programs that address } \\
\text { identified needs } \\
\text { - to monitor or evaluate } \\
\text { programs } \\
\text { - to provide information for } \\
\text { advocacy for policy change } \\
\text { - to test hypotheses as part of } \\
\text { scientific research }\end{array}$ & $\begin{array}{l}\text { - surveys or questionnaires } \\
\text { - in-depth interviews } \\
\text { - observations } \\
\text { - focus group discussions } \\
\text { - personal histories through } \\
\text { - storytelling or diaries } \\
\text { - rapid assessments } \\
\text { - participatory methods (e.g., } \\
\text { mobility mapping, pile sorting) } \\
\text { - community meetings } \\
\text { - examination of case records } \\
\text { - testing of biological samples }\end{array}$ & $\begin{array}{l}\text { - children and adolescents } \\
\text { - adult family members, } \\
\text { caregivers } \\
\text { - community members } \\
\text { - peers } \\
\text { - case records (e.g., medical, } \\
\text { school) } \\
\text { - adults working with children } \\
\text { and adolescents (e.g., } \\
\text { teachers, health workers) }\end{array}$ \\
\hline
\end{tabular}

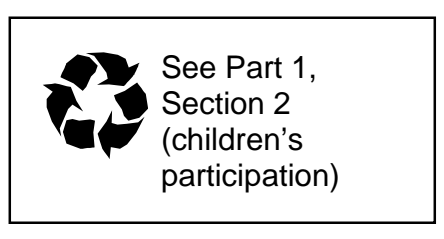

In this publication, the most important principle is to al ways put the best interests of the child first by promoting and protecting their well lbeing. At a minimum, programmers and researchers collecting information must do no harm. This publication suggests ways to implement these principles and to protect children and adolescents.

A nother important principle expressed throughout this publication is the value of children's participation. Children and adolescents are often the best sources of accurate information about their own lives. Their perspectives on decisions about their care and their future are important. They have a right to express their views about decisions affecting their own lives and those of their families or communities. 


\section{Part I - Key Considerations}

This section identifies crosscutting themes that underlie all the practical guidance that follows. It presents principles and safeguards to ensure that information gathering among children and adolescents is done ethically at all stages of the activity.

\section{Basic ethical principles}

Strict observance of ethical principles is especially important when working with children and adolescents because of the difference in power between the participant and investigator.

These guidel ines for information-gathering activities are informed by fundamental principles of medical research. Although they were originally developed for biomedical clinical studies, their ethical implications rise above all geographic, cultural, economic, legal, and political boundaries. These ethical principles also apply to program managers implementing activities to monitor and evaluate programs and to social and behavioral researchers.

The widely accepted ethical framework used here presents three fundamental duties of the investigator: respect for persons, beneficence, and justice. ${ }^{1}$ Strict observance of these responsi bilities addresses the difference in power between participant and investigator that leaves the child or adolescent vulnerable to exploitation.

\section{Respect for persons: A competent individual has the right to self-determination.}

(a) Individuals should be treated as autonomous agents.

"An autonomous person is an individual capable of deliberation about personal goals and of acting under such deliberation. To respect autonomy is to give weight to the autonomous person's considered opinions and choices while refraining from obstructing his or her actions...." 2

This principle addresses the need to seek individual informed consent. Prospective participants must have as much time and information as they need to decide whether or not they wish to participate in an information-gathering activity. They must be able to make an independent decision without any pressure.
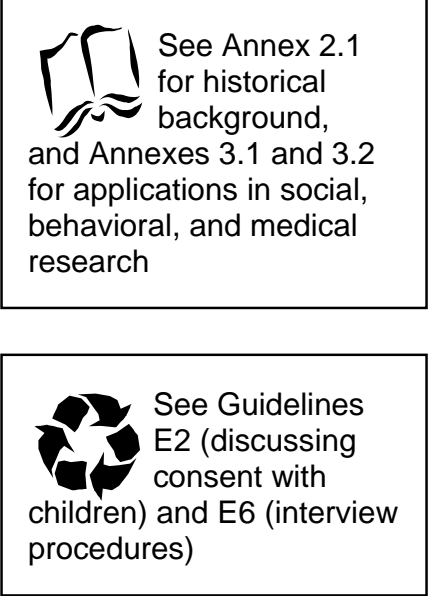

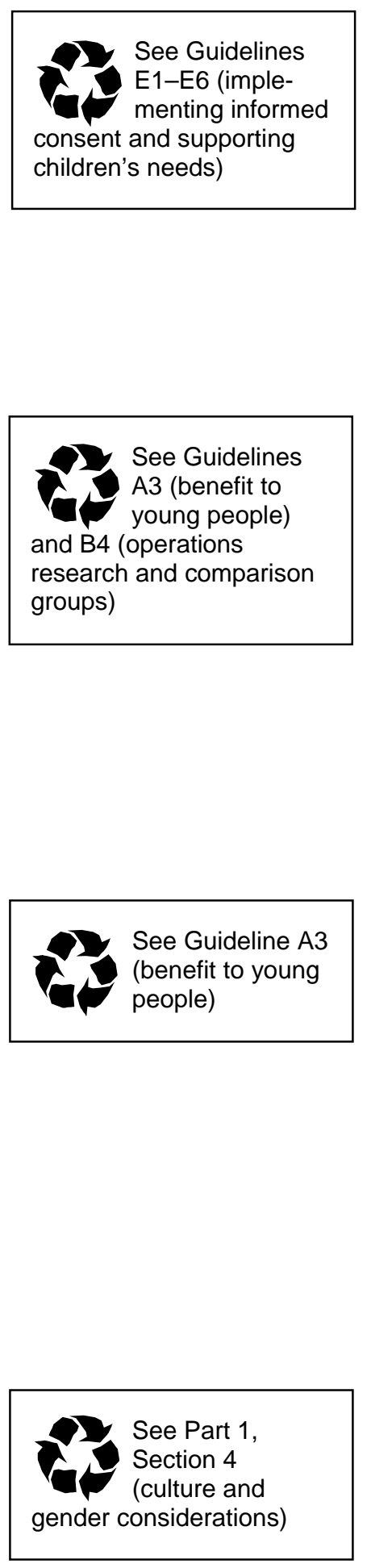

(b) Persons with diminished capacity need additional protections.

Special arrangements are required when a person's understanding is limited due to age or devel opmental disabilities. Children's capacity to make and understand decisions develops as they grow up. They must have the opportunity to express views and make choices in a way that is appropriate to their understanding. If children are under the legal age of consent, permission is required from their parents or legal guardians.

\section{Beneficence: An investigator must protect participants from harm and maximize possible benefits.}

This principle means that the investigator is responsible for the benefit of participants during the information-gathering activity, making sure they receive maximum benefit while being sure to do no harm. It is often a challenge to determine the right balance between benefits and harm. The goal of gathering information is to benefit society, but this does not justify causing serious distress or injury to any individual. The investigator must do everything possible to anticipate potential negative consequences and ensure that the activity will not lead to direct or indirect harm.

\section{Justice: The benefits and burdens of the activity should be equitably distributed.}

The principle of justice requires investigators to consider the balance between risks and benefits among participants. Participants must be treated fairly, and the risks and benefits of the activity must be distributed equitably and without bias. Information-gathering activities should not involve persons who are unlikely ever to benefit from the findings.

The concept of justice must be considered when deciding who will be given an opportunity to participate, who will be excluded, and the reasons for exclusion. The investigator should select participants fairly in relation to the aims of the activity, rather than simply because of their availability, their compromised position, or their vulnerability.

Like the social world of adults, the social world of children can be uneven; within groups of children there are different levels of power. Following the principle of justice, investigators should ensure that information-gathering activities are designed to include input from a variety of children and adolescents who can represent the distinct realities of these more powerful and less powerful groups. 


\section{Children's participation}

\section{Children must have the opportunity to express their views about activities that affect their welfare, and these views should be respected.}

In order to adhere to the basic ethical principle of respect for persons, children and adolescents must be treated with honesty and integrity. Their opinions provide a valuable perspective that should be respected and responded to in an age-appropriate way. Investigators have a responsibility to identify appropriate opportunities and roles for young people to express their views about the information-gathering activity in ways that are appropriate to their age.

Children and adolescents can provide crucial information about their needs and how to respond to them. For example, they can review questionnaires and advise investigators if the proposed techniques for gathering information are appropriate or likely to be reliable, or if any questions might cause distress and should be changed. They can also help to collect information from other children and assist with the interpretation and communication of the results.

Children's participation helps improve methods for gathering information, thus enhancing the accuracy and usefulness of the findings. Involving children in age-appropriate decision-making may also have other benefits, including providing opportunities to improve their self-esteem and support of each other. Promoting the participation of children and adolescents recognizes their potential to enrich decision-making processes, to share their perspectives, and to participate as active citizens.

In addition, it is unethical to prevent children and adolescents from participating in decision-making about things that affect their lives. The principle of children's participation affirms that children and adolescents have the right to express their views in all matters affecting them. It requires that their views be heard and given due weight according to their age and maturity, in accordance with human rights conventions. Participatory approaches must also be designed to ensure that information gathering supports inclusion of minority voices, such as those with disabilities, is nondiscriminatory, and is ageappropriate.

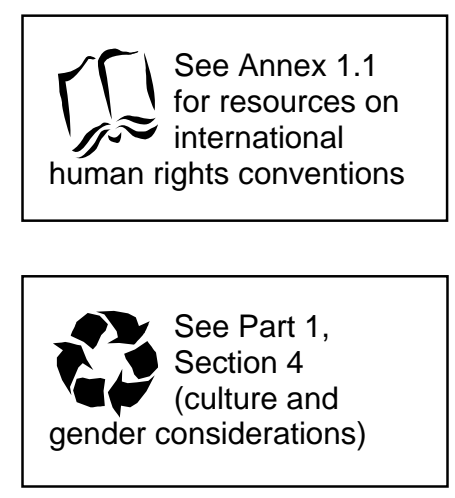



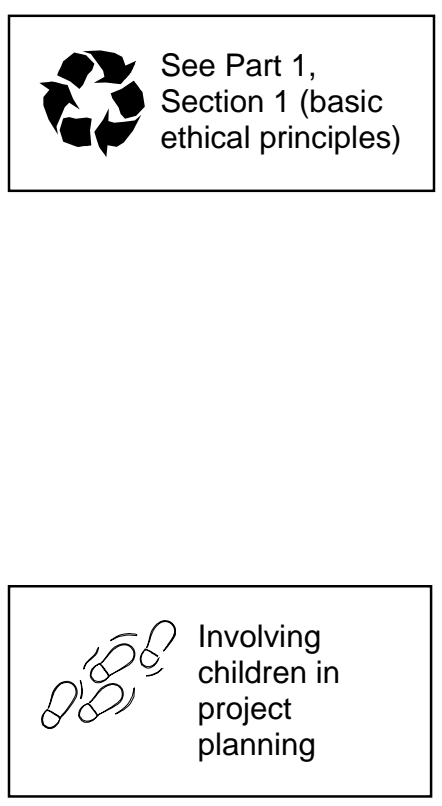

Investigators must pay careful attention to balancing the two distinct roles that children and adolescents may play in the informationgathering activity:

(a) Children and adolescents as participants.

Participatory approaches that promote the inclusion of input from children and adolescents in the design and development of information-gathering activities are an important way to recognize young people's views and should be used to the maximum extent possible.

(b) Children and adolescents as respondents.

Information gathering on sensitive topics can be upsetting and should be regarded as intrusive. To protect children from harm, the use of information-gathering methods should be kept to the strict minimum required to gain appropriate information on sensitive issues, in accordance with the basic ethical principle of beneficence.

Giving children and adolescents the maximum opportunity to express their views must be balanced with protecting their best interests and safeguarding them from potential harm by minimizing intrusion. Children and adolescents must have the opportunity to express their opinions without compromising their safety and well being.

"In M bale District, in U ganda, we asked children ages 5-10 years about their perceptions of poverty; for example, what made someone poor, how you could tell that they were poor, and why they were poor. W e discussed these questions with groups of children from mixed backgrounds and groups of children that teachers knew were more vulnerable. The children yielded very rich answers, which we used for planning the next stages of the community development projects.

"Besides getting the ideas of the children themselves, one of the best results of this exercise was the impact on the staff. I don't think they realized how much children of this age could contribute and how much they understood. Children and youth tended to mention more of the socially challenging causes (abuse, drinking, neglect) than adult respondents. A s a result of the children's in put, these causes are also addressed in some aspects of the programs that are being developed." Program manager, U ganda 
"We, the children, are experts on being 8, 12, or 17 years old in the societies of today.... To consult us would make your work more effective and give better results for children. M y proposal is that you make us part of your team." Girl, age $17, \mathrm{~N}$ orway

"U sually when we children talk about participation and making our views heard, most adults see it as a passport to disrespect. The adults have forgotten that the world is dynamic and things we know now they didn't at our age and might still not know. We have to move from the stage where children are to be seen and not heard to a stage where children should have a voice in decisionmaking in matters that affect him or her." Girl, age 14, G hana

\section{Legal and professional requirements}

\section{It is the investigator's responsibility to make sure that local, national, and international legal regulations and accepted ethical standards for the conduct of the activity are met. Information- gathering activities require ethical supervision mechanisms to independently protect the best interests of children and adolescents.}

The conduct of staff who gather information from children and adolescents is governed by both legal and ethical requirements. Adults working with children may be subject to multiple legal codes governing their behavior. These codes may include those of the country of the organization managing the activity, the country funding the activity, and the country where the activity is taking place.

Investigators should strive to meet the highest possible standards among these codes. It is particularly important to make sure that local laws are followed and local definitions are used, especially for the process of gaining informed consent. (For example, the age of majority in most European countries and the United States is 18 years; in Swaziland, 21; in India, 18; and in A Ibania, 14.) National regulations may require permission from or regulation by local scientific institutions. Where local laws are unclear or where local customs are in conflict, refer to the basic ethical principles outlined above, and remember that preserving the safety and protection of the child is the most important value.

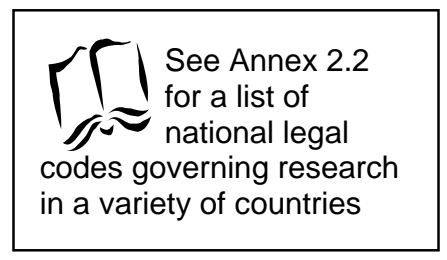


Some professional groups, such as social workers, psychologists, sociologists, and anthropologists, have established codes of ethical standards that govern their work with young people. Even when the adults working with children are not formally bound by such codes, they provide a useful guide for conduct by establishing professionally accepted standards.

Examples of questions that require attention to legal and professional implications:

? Who has authority to grant permission for access to children and adolescents as participants?

? What will happen if the child or adolescent reveals information indicating legal or illegal activities that could bring harm to the child, family, or community?

? What is the responsibility of researchers who uncover serious problems or needs, such as abuse, neglect, or malnutrition?

\section{Ethical supervision}

Ethical supervision is an important part of the responsible and ethical conduct of activities to gather information, and is often legally required before starting an information-gathering activity. An ethical supervision mechanism is used to oversee the design and conduct of activities to protect young participants from harm. Whether the results will be used for research purposes or program evaluation, the impact of questioning children and adolescents is similar. In each type of activity, comparable safeguards are required to protect children, although different mechanisms may be appropriate.

The organization providing funding for an activity bears the major responsibility to ensure that its funds are used ethically. They must establish regulations for ethical supervision by requiring plans for the activity to be reviewed by an in-country ethical review board where possible, or by establishing in-house ethical review mechanisms. In addition to the professional expertise provided by an ethical review board, use of a community advisory board is also recommended.

Ethical review boards

For research activities, most national legal regulations require the research institution (such as a university or non-profit research 
organization) to establish an independent ethical review board (also known as an ethics committee) to thoroughly review all research plans (protocols). Research activities cannot progress without approval from an ethical review board. Ethical review boards are often required to include community representatives.

Program monitoring and evaluation activities often are not legally bound by the strict requirements of formal ethical review that apply to research. The lack of a legal requirement, however, does not relieve the program manager of the responsibility to ensure that informationgathering activities are carried out ethically. Program managers should strive to uphold the same high standards of formal ethical supervision that apply to research activities, especially when the protection of children and adolescents is at stake.

Where in-country mechanisms exist for ethical review, they should be used. Reviewers must be familiar with the local context of children's circumstances, including social and cultural issues. Sometimes going through an in-country review is required by law. For example, a research activity in Uganda requires formal approval from the Ugandan National Council for Science and Technology.

Where in-country review mechanisms do not exist or lack expertise in working with children, review by an alternate institution may be required. However, the investigator may be able to use the opportunity to create or improve the in-country mechanism for review of children's activities; e.g., working with the social affairs or child psychology unit of a university.

Community advisory boards

Community advisory boards (also known as local stakeholder groups, community review boards, or advisory committees) provide an opportunity for investigators to consult with communities. These groups provide a mechanism to address community perceptions of the proposed activities, assessing risks and benefits and ensuring children's protection throughout the activity.

Supervision and feedback mechanisms used for monitoring and eval uation and research activities are compared in Box 2. Modification to these methods may be required for other types of informationgathering techniques, including rapid assessments in emergency situations and small-scale activities.
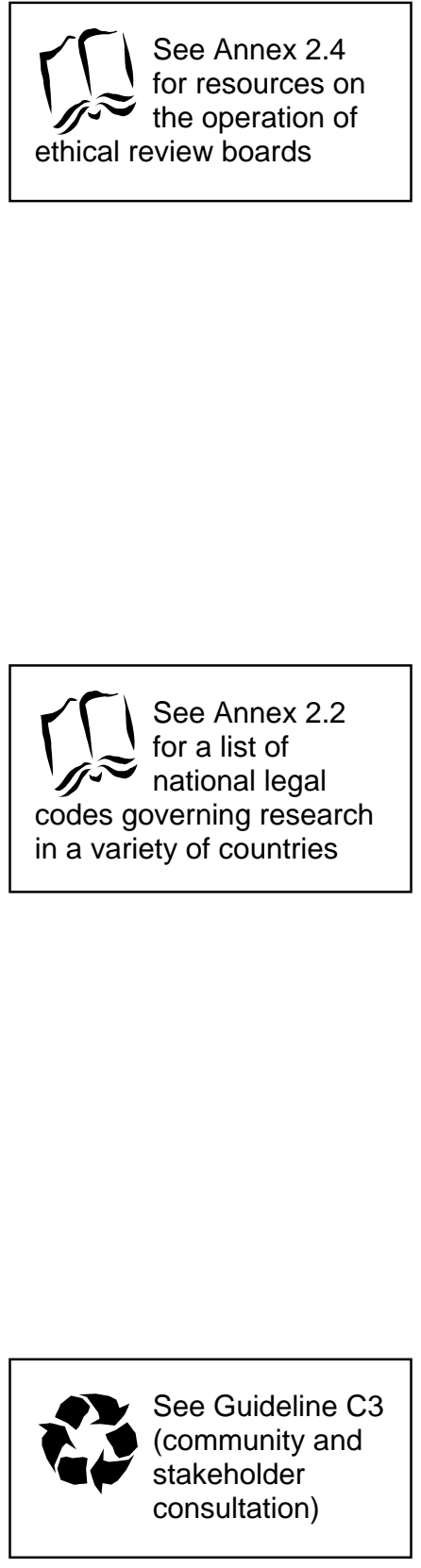
Box 2 Supervision and feedback mechanisms

\begin{tabular}{|c|c|c|}
\hline & Ethical review board & Community advisory board \\
\hline Purpose & $\begin{array}{l}\text { To ensure that activities adhere to accepted } \\
\text { legal and ethical practice and scientific conduct }\end{array}$ & $\begin{array}{l}\text { To ensure that activities are locally understood } \\
\text { and acceptable, in accordance with ethical } \\
\text { standards }\end{array}$ \\
\hline $\begin{array}{l}\text { Required for } \\
\text { program } \\
\text { monitoring and } \\
\text { evaluation? }\end{array}$ & $\begin{array}{l}\text { - Not legally required, but may provide a } \\
\text { useful guide }\end{array}$ & $\begin{array}{l}\text { - Ethically recommended } \\
\text { - May also be mandated by institutional } \\
\text { regulations or donor organizations }\end{array}$ \\
\hline $\begin{array}{l}\text { Required for } \\
\text { research } \\
\text { activities? }\end{array}$ & $\begin{array}{l}\text { - Legally required (check national and } \\
\text { international regulations) }\end{array}$ & $\begin{array}{l}\text { - Ethically recommended } \\
\text { - May also be mandated by institutional } \\
\text { regulations or donor organizations }\end{array}$ \\
\hline Composition & $\begin{array}{l}\text { - Experts of various backgrounds with } \\
\text { knowledge from working in relevant } \\
\text { activities } \\
\text { - Members with expertise in child } \\
\text { development } \\
\text { - Members representing minorities and } \\
\text { gender balance } \\
\text { - Qualifications and conflicts of interest must } \\
\text { be openly documented } \\
\text { - } 5 \text { member minimum (U.S. regulations only) }\end{array}$ & $\begin{array}{l}\text { - Local community members representing } \\
\text { children, selected from local community and } \\
\text { organizations involved in or affected by the } \\
\text { activity } \\
\text { - Members with expertise in child } \\
\text { development } \\
\text { - Members representing minorities and } \\
\text { gender balance } \\
\text { - Qualifications and conflicts of interest must } \\
\text { be openly documented }\end{array}$ \\
\hline $\begin{array}{l}\text { Participation of } \\
\text { children and } \\
\text { adolescents }\end{array}$ & Unlikely & Highly recommended \\
\hline $\begin{array}{l}\text { Role before } \\
\text { granting } \\
\text { approval }\end{array}$ & $\begin{array}{l}\text { - Ensure that children and adolescents are } \\
\text { protected from harm } \\
\text { - Withhold approval if protection is not } \\
\text { assured }\end{array}$ & $\begin{array}{l}\text { - Ensure that proposed activities are } \\
\text { appropriate according to children's age and } \\
\text { culture } \\
\text { - Ensure that children and adolescents are } \\
\text { protected from harm } \\
\text { - Withhold approval if protection is not } \\
\text { assured }\end{array}$ \\
\hline $\begin{array}{l}\text { Role after } \\
\text { granting } \\
\text { approval }\end{array}$ & $\begin{array}{l}\text { - Review any changes to protocol, informed } \\
\text { consent form, study sites, or recruitment } \\
\text { procedures, and any operational or safety } \\
\text { problems encountered } \\
\text { - May conduct annual reviews of ongoing } \\
\text { activities }\end{array}$ & $\begin{array}{l}\text { - Hold meetings during the planning process } \\
\text { and regularly throughout the duration of the } \\
\text { activity to monitor continued protection of } \\
\text { children and adolescents, monitor } \\
\text { acceptability of activities, and respond to } \\
\text { issues that arise }\end{array}$ \\
\hline $\begin{array}{l}\text { Special } \\
\text { interests and } \\
\text { roles }\end{array}$ & $\begin{array}{l}\text { - Require detailed examination of the } \\
\text { informed consent process }\end{array}$ & $\begin{array}{l}\text { - Provide culturally specific input into } \\
\text { assessment of risks and benefits for } \\
\text { participants and communities } \\
\text { - Develop child protection procedures and } \\
\text { informed consent process }\end{array}$ \\
\hline
\end{tabular}




\section{Culture and gender considerations}

Investigators should consult with local advisors to make sure that the activity is culturally acceptable. They should also ensure that a gender-sensitive perspective informs the planning, implementation, and analysis of activities, taking into account the role of gender inequality and power imbalance in the ability of boys and girls to participate and the risks they face.

To provide maximum protection to young participants, investigators must be familiar with and respect their cultural norms, including age and gender roles within the family and community, limitations on social roles imposed by ethnicity or social group, and expectations for child development and behavior. The input of local community members is crucial to developing the activity, minimizing harm to participants throughout the activity, and ensuring accurate interpretation of the results.

Setting up a community advisory board, as outlined in the previous section, provides an important mechanism for investigators to receive feedback from community members about the acceptability of activities.

Examples of questions about culture that affect informationgathering activities:

? How do community elders respond when children speak out in their presence?

? To what extent does an adolescent feel personally empowered to agree to participate in an activity?

? How will children of different ethnic groups or poverty levels interact in a group information-gathering activity?

Making sure that activities are conducted in a gender-sensitive manner is one of the most important cultural considerations. Gender sensitivity requires investigators to question the ways in which the relative status of males and females in the society under examination will be reflected in the activity. This includes the way information is gathered and the way the findings are presented and used. Examining methodologies and results with a keen awareness of the dynamics of gender will help investigators analyze attitudes and identify biases and social conditioning. 
Examples of questions that reflect a gender-sensitive perspective:

? What are the expectations regarding school attendance and household responsibilities for girls and boys following the loss of a parent?

? How will community members regard a young female participant speaking alone with an older male interviewer?

? What social pressure does a young male participant face when revealing personal emotions that might cause him to cry?

\section{Considerations for especially vulnerable children}

\section{Children who are in especially vulnerable situations require additional safeguards to protect their welfare.}

Maintaining ethical standards is particularly important for children and adolescents without a parent or guardian, or those who are not protected by an effective legal system because of displacement, refugee status, or social marginalization. Approval from a child's parent or guardian is required whenever possible, but many vulnerable children and adolescents may not have a legally responsible adult to look after their interests.

Orphaned, separated, and unaccompanied children and adolescents require special considerations to avoid being exploited. For example, in the United States, children and adolescents who have been separated from their parents and close family members, including those in the care of agencies, may be included in a study only if it is:

- $\quad$ specifically related to their status as separated children; or

- $\quad$ conducted in schools, camps, hospitals, institutions, or similar settings in which all children are included as participants. ${ }^{3}$

Children and adolescents living in circumstances of armed conflict, severe poverty, famine, or other destabilizing events face increased risks with no guarantee of safety. A dults working with children and adolescents in these particularly insecure settings, where infrastructures are weak or populations are mobile, face many challenges when collecting accurate information. For example, it is 
often harder to provide follow-up services to meet children's needs, and the information revealed may be particularly sensitive.

Program managers struggling to meet the basic needs of war-affected populations may be unable to perform even routine monitoring and evaluation of their services. In such circumstances, investigation teams must be extremely sensitive to the nature of their activities and question whether it is appropriate to contact children. When it is possible to enter such communities, investigators face the difficult task of anticipating consequences that threaten the safety of children, their families, and their communities. This requirement extends throughout the activity, including attention to collecting data anonymously or keeping records of disclosed personal information confidential long after the activity is complete.

\footnotetext{
${ }^{1}$ This section is adapted from the National Institute of Health online training course in Human Participant Protections: Education for Research Teams http:/ / www.cancer.gov/ clinicaltrials/ learning/ page3

2 National Commission for the Protection of Human Subjects of Research. Belmont Report: Ethical Principles and Guidelines for the Protection of H uman Subjects of Research. U.S. Department of Health, Education, and Welfare, Office of the Secretary, 1978. (Belmont Report)

${ }^{3}$ Code of Federal Regulations: Title 45 - Public Welfare Department of Health and Human Services: National Institutes of Health/ Office For Protection From Research http:/ / ohrp.osophs.dhhs.gov/ humansubjects/ guidance/ 45cfr46.htm\#
} 
Part 1 - Key considerations

14 Ethical Guidelines for Gathering Information from Children and Adolescents 


\section{Part 2 - Practical Ethical Guidelines for Information Gathering}

This section provides a practical guide for the implementation of ethical standards, the fundamental tools for protecting children and adolescents. There are no clear answers to the challenges listed below, only guiding principles to enable each case to be analyzed on a practical basis, with the primary principle being the protection of the children or adolescents.

These guidelines are presented as questions and responses within six subsections:
A. Is this activity necessary and justified?
B. Is this activity designed to get valid information?
C. Have community and stakeholder concerns been considered?
D. Have adverse consequences of the activity been anticipated?
E. Are procedures in place to ensure that the activity proceeds ethically?
F. Is there a clear plan and adequate funding for follow-up activities?

\section{A. Is this activity necessary and justified?}

This section examines the questions that all investigators must consider before deciding to proceed with any activity.

\section{A1 Is the purpose of the activity clearly defined, and are sufficient human and financial resources available to conduct it in an ethical manner?}

\section{Before starting the activity, identify its intended goals, audience, and the staff and money needed to conduct it ethically, and confirm with all stakeholders.}

Activities to gather information from children about their lives should be developed in the context of an ongoing programmatic approach with high levels of child participation. Without this approach, gathering information directly from children and adolescents should be regarded as a disruptive intrusion into their lives. Risks to children and adolescents include raising expectations or causing other 
inadvertent harm. With this warning in mind, investigators must have clear answers to the following questions before proceeding:

- Who needs this information?

- What is this information needed for?

- How much money is available to conduct this activity?

The activity should only proceed if the practical requirements of the information users can be balanced with the ethical treatment of the children or adolescents who will be involved. Ethical treatment requires the use of appropriate methods that respect children and adolescents, and sufficient funding to implement the required procedures documented in these guidelines.

A2 Is the required information available elsewhere? D oes it already exist in documents or can it be gathered from older informants?

\section{Only use direct methods with children if the required information is not available any other way.}

Whether it is necessary or appropriate to gather information directly from children or adolescents depends on:

- their ages and developmental stages;

- their ability to express opinions and feelings about their experiences; and

- whether the required information is potentially upsetting in nature.

In situations where younger children might be more vulnerable to distress, investigators should consult with children and community members to consider carefully how to ask age-appropriate questions. They also should consider whether the required data could be more sensitively and usefully collected from older children and adolescents.

as respondents.

When possible, survey information should be obtained from an alternative indirect source, such as talking to adults (family, caretaker, or community members) close to the child or examining written records. For example, health records may provide information on a child's immunizations. 
If the required information is already available from alternative sources then the interference with children's lives cannot be justified. Multiple rounds of information gathering to determine trends over time should be kept to a minimum. Individuals and communities subjected to repeated inquiries become understandably frustrated and wary of such intrusions, especially when they see limited personal benefit.

\section{A3 Will the young people involved in this activity benefit from it?}

\section{If the information-gathering activity will not directly benefit the children and adolescents involved or their community, do not proceed.}

The children and adolescents taking part in the information-gathering activity must receive some direct, immediate benefit from their involvement. It is harder to ethically justify a long-term project with no immediate benefit for the children. Investigators should make every effort to link research studies with programs and services that provide benefits to justify the potentially harmful intrusion into children's lives and prepare to respond to the individual circumstances that might be revealed. Children and adolescents should be protected from information-gathering efforts that will not benefit them directly. The ethical justification of descriptive, exploratory, or diagnostic research and long-term academic investigations raises many questions.

If an explicit and immediate link to programmatic services is not possible, then the participants should gain some other benefit from their involvement. For example, participants of formative research to design a program addressing the psychosocial needs of vulnerable children, or participants in a long-term anthropological study of children involved in sex work that will be used in advocacy, might not gain immediate benefit from these activities. However, investigators can make sure that children participating receive some other direct and immediate benefit, such as access to on-site counseling or complementary services. This requirement should not be used to justify incentives rewarding participation. 

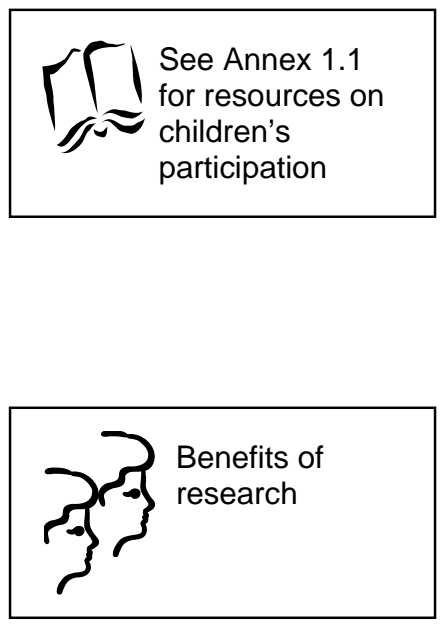

Children and adolescents who actively participate in an informationgathering activity may be personally empowered by this process, but these methods must be used carefully. They do not simply justify discussing difficult subjects. The anticipated benefit to participants should be linked to the intrusiveness of the questioning permitted. Asking potentially difficult questions that require follow-up support may be appropriate only as a part of the monitoring of an alreadyexisting program that provides or facilitates access to services.

"Research on children raises particular ethical issues because the subject nature can be extremely sensitive and the effect on children's emotional health can be considerably different than [the effect of] research questions asked to adults. It is important that the information not be collected just because we want to know something, but because there is an application in sight that will have direct influence on programming and policy development leading to improved interventions for children. This has become my standard statement to anyone wanting to carry out research with children." R esearcher, Zambia

\section{B. Is this activity designed to get valid information?}

It is unethical to involve children in an activity that will not result in valid information. This section describes some of the procedural safeguards required to ensure the collection of valid and useful data. However, this is not intended to provide a comprehensive methodological guide to the practical requirements of assessing the situation of young people.

\section{B1 Is there an agreed description of how the information will be collected, analyzed, and used?}

\section{D evelop a protocol to clarify aims and procedures to which partners agree.}

All partners involved in the information-gathering activity must be in complete agreement about its conduct before it begins. Investigators should develop a protocol stating the objective, design, methodology, and management of the information-gathering activity. This document must be available for review by all staff, community members, and other interested parties at any point during the activity. Community members should have the opportunity to review and provide feedback on the protocol, and this feedback loop must be respected. 


\section{B2 Does the sampling methodology ensure that all children have an equal opportunity to participate, recognizing gender, ethnicity, and other barriers?}

\section{A pply community definitions to set clear criteria for inclusion. Use existing records when possible, and recognize social and cultural barriers to participation. For surveys, use a minimum number of respondents to achieve demonstrable results.}

Investigators should define clear inclusion and exclusion criteria for child and adolescent participants, in discussion with community members. Locally accepted definitions, including ethnicity, socioeconomic status, and vulnerability, should be used. Minimum age requirements should be determined in consultation with community members. These criteria will determine who is involved in the activity and the extent to which results can be viewed to represent a particular group.

Where other local agencies have already identified appropriate children and adolescents, for example, to provide services, investigators should use their definitions. Where possible, they should work through their pre-existing structures to gain access and consent to work with these young people, to avoid the possibility of further stigma.

The investigators should consult with local experts to make sure that the selection of participants includes representation from minority groups. The views of children who face social barriers may differ from the majority in an important way. For example, interviewers may have to make special efforts to include the views of young women in a society where they are not traditionally encouraged to speak out.

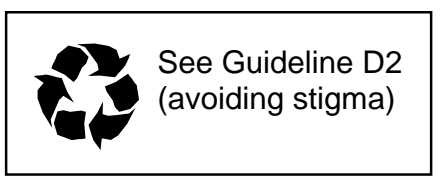

The use of age-appropriate survey methods, such as questionnaires, must be carefully planned to reach a group of young people of the appropriate size and composition, while minimizing intrusions into the lives of children and adolescents. When deciding how many respondents to include in a survey, investigators must balance the conflicting pressures to approach more individuals to achieve reliable results, and to approach fewer individuals to minimize the intrusiveness of the activity. Some quantitative approaches are unsuitable for use with young children; the input of community members is crucial to determining age-appropriate methodological approaches. Investigators should use the minimum number of respondents required to give valuable results. For a quantitative 
research study this may be determined by using a statistical calculation. ${ }^{1}$ Involving too many young respondents cannot be justified if it will disrupt their lives. Yet involving children and adolescents in an exercise that has too few respondents to produce the intended results is also unethical. Investigators planning an information-gathering activity must anticipate the likelihood of missing information if respondents decide not to answer certain questions, and must verify that the number of responses required to provide meaningful results is still achieved.

\section{B3}

Have tools for gathering information been developed with qualified professionals? Have the tools been locally translated and field-tested?

\section{D evelop all tools, such as questionnaires, through discussions with experts. These tools should then be translated locally, back- translated, and field-tested.}

The methods and tools used when working with children, such as questionnaires, focus group discussion guides, and visual aids, must be designed specifically for the needs of the group, considering their age and culture. They should be developed in cooperation with professional experts in child development, and informed by discussions with the children themselves and with adult community members.

Ideally, a local native speaker should translate all materials into the local language. The translation should then be back-translated into the original language by an independent local translator to verify its accuracy, confirm understanding of key concepts, and prevent misunderstandings.

Before the tools are widely used, they should be pre-tested among a small group of children who resemble the study population in terms of their age, sex, ethnicity, and socioeconomic status. Field-testing activities with a small sample of children provides the opportunity for investigators to identify potentially negative consequences and modify the planned activities accordingly. The feedback from this process must be respected and acted upon. Repeated field-testing of modified activities should be conducted, if necessary. 


\section{B4}

\section{Is the use of a comparison group for evaluation purposes} appropriate? Can it be ethically justified?

\section{Use comparison groups only under careful ethical supervision. A comparison group totally deprived of services is inappropriate with vulnerable children. In such cases, alternative approaches should be explored to strengthen research findings.}

Traditional program evaluation is frequently done without the use of a comparison group. However, more rigorous research to test the effectiveness of a new program generally involves comparing those who receive the program to a comparison group that is not exposed to the program. This approach, which originated in clinical randomized controlled trials for pharmaceuticals, is sometimes used in program evaluation and operations research because it strengthens the ability to demonstrate the program's effect clearly.

Considering the use of comparison groups reveals tension between methodology and ethics. On one hand, investigators aim to demonstrate that a program really works through a robust evaluation. On the other hand, investigators are ethically required to provide services for the maximum benefit of all the children that they work with, in accordance with the principle of beneficence.

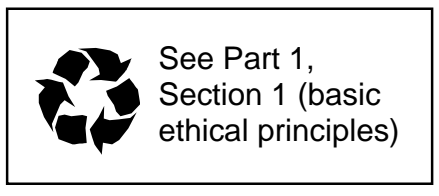

The requirement to treat participants ethically is always more important than the scientific requirements of research methodology. It is almost always unethical to test an experimental program by monitoring over time a group who is not receiving the benefits of that program, unless the benefits of that program are truly unknown. In clinical trials, control groups may be used to test the effects of new medications when their effectiveness is truly unknown. But when piloting new programs for highly vulnerable children, this sort of research is extremely inappropriate. When the question being investigated is not whether to offer services at all but how to offer services, using a comparison group with no access to services is almost always unethical. Donors and program implementers alike must keep this in mind when they seek empirically-proven best practices.

Alternative ways to strengthen the design of a program evaluation or operations research study to get an indication of program impact without using comparison groups include:

- conducting a baseline survey before an intervention begins;

- observing the program in multiple settings; 
- observing the program at different levels of intensity; and

- using multiple methods to assess program impact, such as combining quantitative and qualitative techniques and observations.

If these alternatives are not possible, another way to keep the evaluation rigorous without compromising ethical standards is to find a natural comparison group that already exists. For example, program implementers often phase in their services, starting in one geographic region and gradually expanding and fine-tuning services as more funds become available. Under these circumstances it may be possible to work with a comparison group that will not have access to the initial phase of services, but will have access to the expanded and hopefully improved services later when they are offered in their area.

"We conducted a study of young people receiving services through a psychosocial support program. We identified a similar group of young people in a neighboring community that had not received the services, which we used as a comparison group. We arranged with the program implementers that every youth participating in the survey in the comparison group would be guaranteed enrollment in one of the experimental support programs immediately after the survey. So in this case the research actually led directly to services for a group of youth who might not have received those services otherwise.

"The study would have been more rigorous if it we had been able to return to conduct repeated surveys with the intervention and comparison groups, but we could not ethically justify withhol ding services from the comparison group for any period of time." Researcher, $U$ ganda

“W e wanted to test an innovative program for orphans and vulnerable children in $U$ ganda. W earranged with the program implementers to survey a comparison group at the same time as the intervention group in an area where the program was to be phased in later. A s it turned out, the implementing organization was unable to offer services to the comparison group during the course of the study. Some of the researchers were tempted to return to the comparison communities to continue data collection as originally planned because this information would provide powerful results. But our interviewers, who had already been in contact with the community during the first survey round, felt strongly that this would be inappropriate. It would raise expectations about the services that were not going to arrive, and the long and potentially intrusive interviews with children could no longer be ethically justified. At that point in the study, we knew that the use of a comparison group had become unethical, and we ended activities in that area." R esearcher, U ganda 
"W e piloted a new training course for youth club members on providing care and support to people living with HIV and AID S. For ethical reasons, we knew that we could not simply compare one group of young people receiving the training course to an equival ent group receiving no training at all. So we used a comparison group who received a training course on HIV prevention only, to the best standard locally available. W e compared the effects of our en hanced training package to the standard prevention-only training." Researcher, Zambia

\section{Have community and stakeholder concerns been considered?}

Local community members and interest groups are the most important informants about the acceptability of activities within their area. Investigators are responsible for seeking out and appropriately recognizing the views of local groups as they plan and implement their work with children and adolescents.

\section{C1 Have all appropriate community and related groups with an interest in the results been consulted for permission to work with children?}

\section{Consult locally to determine who must give permission for the activity to proceed.}

As investigators anticipate the levels of permission required to protect children and adolescents in the information-gathering activity, they must consider the different levels of adult and community decisionmaking authorities. Before commencing the information-gathering activity, investigators must consult with local stakeholders with interests in children or adolescents' wellbeing to seek guidance in accordance with cultural variations in understanding of autonomy and responsibility.

Local law or cultural expectations may require the approval of local authorities, community elders, or other so-called gatekeepers.

Permission to approach children also may be required from other adults, in addition to the formal legal requirement of consent from a parent or guardian. Asking community groups for their input on the proposed activity is necessary and respectful. Involving community See Guideline E1 (who gives consent) groups has the added advantage of giving investigators an 
Community gatekeepers: Whose approval is required? opportunity to seek wider support and visible endorsement from locally respected figures.

"W e recently conducted a nationwide study on the coping strategies of children to better understand how communities, organizations, and institutions can support orphans and vulnerable children. O ne of our main steps was to develop protocols for gaining permission to work with children. W ith our partner organizations we developed a register of people and institutions that researchers had to approach for permission before entering a community to work with children. The list included government agencies, schools, health centers, the police, political and religious leaders, and other local organizations.

We trusted our partners to know best with whom they would need to make contact to ensure that information about the study would filter down through existing structures in their catchment areas. Formalizing the required communication channels hel ped our partners create additional linkages. Identifying and involving these gatekeepers also made the task of following up with at-risk children easier, because the rel evant stakeholders had been involved in the research initiative from the beginning. This process al so created vital outlets for releasing our findings. W e have been able to share our results with the community members we identified as gatekeepers, acknowledging the importance of their role and enabling them to be better informed for decision-making at the community level." Researcher, Zimbabwe

\section{C2 Have efforts been made to ensure that the community and potential participants understand the activity's purpose and possible outcomes to avoid raising false expectations?}

\section{Interviewers must be highly sensitive to the fact that their presence in the community may be highly visible and a source of local interest. Clarify roles and expectations through community meetings, and visibly honor commitments.}

A team of outsiders arriving in a community in a vehicle, especially one bearing the logo of a well-known organization, may convey a misleading message about their control over resources. Children and adolescents, especially those in deprived circumstances, are particularly vulnerable to raised hopes from the presence of investigators. For example, they might assume the investigators will make immediate changes in the community. It is the responsibility of 
investigators to protect children and adolescents from the disappointment of these raised expectations.

Investigators should publicly clarify their roles and responsibilities and those of the participants. They should define in accessible terms the limits of what can reasonably be expected of everyone involved. The best way of doing this is through public meetings with children, adolescents, and adults that are open to the entire community. These meetings should be used to sensitize everyone involved regarding the nature of the work and what outcomes can reasonably be expected. Any commitments made to children or their families must be honored. Investigators should be sensitive to giving any impression that might be misinterpreted as an expectation. The very presence of outsiders simply gathering information in communities can raise expectations. This is why data-collection activities that include interviews of highly vulnerable populations should be linked with programs whenever possible.

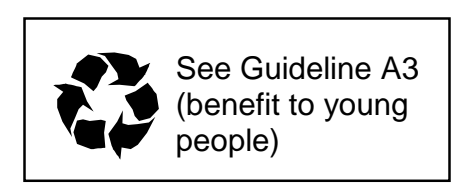

To protect children and adolescents from developing attachments to outsiders who will depart when the activity is over, it may be helpful to train responsible local community members who will remain in the area after the activities conclude to take a more prominent role. Staff conducting interviews and interacting directly with children must balance the need to provide necessary support with the requirement to maintain professional distance so they do not raise undue expectations or create unsustainable attachments.

\section{"O ne of the dangers involved in working with children is that they can} become attached to you and form friendships. If they get to know you and like you, then when you leave their community it may be traumatic for them. They can feel very hurt and betrayed. Even if you honor all the promises, it can still be a wrench when leaving. Therefore researchers must al ways be very honest about how long they are staying and if they are not coming back. Personally, I think researchers must try and keep some distance between themselves and the children they work with to keep from hurting children in the longer term." Researcher, UK

"W e were mobilizing communities in Z ambia to form community committees to support orphans and vulnerable children by providing clothes, food, and educational and housing assistance. In each community we held open meetings where we explained the purpose of our activities. Children in particular provided valuable information to help us to identify meeting participants. They identified key people who were dedicated to improving child welfare and who had helped them individually. Children also described their daily 
experiences to us, enabling us to improve our understanding of the situations they faced as they struggled to survive.

"At the meetings we explained that we were coming into their communities only as facilitators. This point was made crystal clear to all who attended. We explained that the success of this exercise and other project activities depended on full community participation in forming the committees. We emphasized that we were not coming to the communities to give handouts. Community members received, understood, and even appreciated the clarity of this message, which was indicated by the time and effort they dedicated to forming the committees. Community members young and old, men and women, invested their time and became involved.

"Throughout the project, cooperation between the local and district committees that managed the donor funding ensured that the needs identified by the children were honored. For example, the committees stood by their commitment to facilitate recreational activities and provided balls for soccer and netball. This demonstration of commitment enhanced and deepened participation to this program among all stakeholders, including the children themselves." Program manager, Z ambia

See Part 1, Section 3 (legal and professional requirements) for more information on ethical supervision

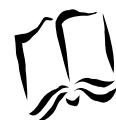

See Annex 1.1 for resources on approaches to working with children and adolescents

\section{A re local supervision mechanisms in place to monitor child protection and review ongoing activities?}

\section{Engage an independent local community advisory group to monitor activities.}

Investigators must ensure the protection of children and adolescents by introducing mechanisms to safeguard them against the misconduct of staff, including intentional and unintentional personal and scientific misconduct. A local group, such as a community advisory group, with independent input from community leaders, teachers, parents, and those responsible for the welfare of children and adolescents, along with experts familiar with language, culture, child development norms, and political circumstances, is an ideal mechanism to monitor the integrity of the activity. The involvement of community members who represent a fair gender and ethnic balance also is important. Children and adolescents should ideally be included in this advisory group. If this is not possible, a separate young people's group should be established.

Investigators are responsible for setting up monitoring procedures to ensure that ethical standards to protect children and adolescents are met and adhered to throughout the information-gathering process. 
Field supervisors should train data collectors in how to implement ethical standards and monitor procedures and results to verify compliance, such as the use of spot checks. Community members or child welfare staff without conflicts of interest in the results should independently verify the supervision and monitoring procedures and results.

\section{Have adverse consequences of the activity been anticipated?}

Investigators are responsible for anticipating direct and indirect consequences of the information-gathering activity as much as is reasonably possible. They should make sure that appropriate safeguards are in place to minimize adverse effects.

\section{D1 Have potentially harmful direct consequences of the activity been anticipated and safeguarded against?}

\section{In partnership with the community, anticipate all possible consequences for the children and adolescents involved. Do not proceed unless appropriate responses to potentially harmful consequences can be provided.}

Having decided to proceed, the paramount ethical consideration is that investigators must do no harm. They must ensure the safety and security of child participants including their physical and psychological welfare as well as their social and communal interests (e.g., exposure to stigma, future prospects for marriage or career). If serious harm appears inevitable even from the least stressful approach to the child, then investigators must conduct a thorough review of alternative sources to find another way to obtain the information, or abandon the activity altogether.

The relative advantages and disadvantages of different techniques for gathering information from children and adolescents must be considered carefully. For example, discussing issues with a focus group including other children in similar circumstances might provide opportunities for children to support each other and share coping strategies. An individual in-depth interview, on the other hand, provides each child with a greater assurance of confidentiality. 

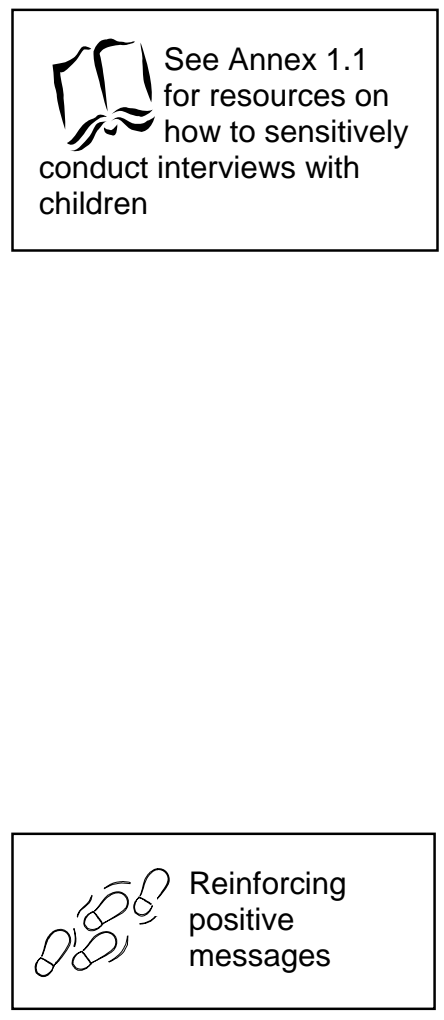

Talking to children about their memories of distressing experiences, such as the loss of a parent or being forced to leave their home during a period of conflict, can provoke strong reactions. Talking about a sad event does not need be an exclusively negative experience, however. As the child recalls traumatic events, for example, a skilled interviewer may be able to make the discussion a beneficial opportunity for the child to express his or her feelings and be heard by a responsible adult. Children and adolescents also might find comfort in knowing that their experiences and perspectives are considered valuable and that they have important information that the interviewer respects and values highly.

Interviewers must genuinely listen to and respect the views of the children and adolescents with whom they talk. They must be sensitive to children's reactions and not override their responses to pursue a line of questioning.

Although interviewers should make every effort to make children feel comfortable, they should also be prepared to respond to the needs of children feeling distress, making referrals if necessary. They must stop the interview if the child becomes upset. Although it might be impossible to be aware of all adverse effects, the investigator should try to anticipate all potential negative repercussions for the child's wellbeing, such as exposure to stigma as a result of involvement, to the greatest possible extent. Children and adolescents may be especially vulnerable emotionally as well as physically during periods of conflict or instability. These risks should be discussed with local community members, including children and adolescents, to determine which kinds of interaction with children are appropriate.

"I have conducted research in the U nited States and Ecuador on children who witness violence. For our study we interviewed 33 boys between the ages of 8 and 12 who had witnessed their mothers being beaten by their fathers. These boys described how they took care of themselves while the violence ensued. Some became tearful. It was very important that the interview end by bringing forward the positive efforts the boys had made to take care of themselves, their siblings, or their pets in that chaos. For all who became tearful, after a pause they wanted to continue. $M$ any said no one had ever asked them about the events they witnessed.

"H ow do we as researchers make sure that children are not left in tears? H ow can we help them gain strength from telling their story and making a difference? H ow can we make sure they have someone else in whom they can confide? I believe part of the answer may be in community participation. We should involve others in the process and conduct of our research. We should 
do more work with groups of children to reinforce the positive in their lives with activities that celebrate them. This means changing our method so that we follow the individual research interviews with some kind of cel ebration or fun creative activity. We can not be simply objective observers. We have additional responsibilities to protect children from painful memories and reinforce the positive in their lives." R esearcher, U S and Ecuador

"D uring our study in Zimbabwe we wanted to have strict guidelines for the interviewers. Even though they were experienced data collectors, they did not have a lot of experience working with respondents from this age group. We trained them to ask a series of questions exactly as they came up in the survey, leaving nothing for interpretation. We could not give the interview ers the flexibility to avoid certain difficult questions because we did not want them to assume they had the freedom to change questions at any time. But what do you do when the protocol requires the interviewer to ask, 'D o you have shoes?' and the child you are sitting with is clearly not wearing shoes?

"Perhaps these difficult issues could have been avoided by designing the survey with better knowledge of the community. In retrospect, I feel we could have paid more attention during our research. We might have been more understanding of the situations of many of the youths and decided not to ask sensitive questions in the way we did. We should have asked more local stakeholders with a real feel for the community to provide in put into the design of the questionnaires and focus group tools. We should have asked if any questions should be avoided, and so on. For example, because our respondents lived in some of the poorest areas of Bulawayo, we could have assumed that some would not have shoes. But first we really needed to consider whether this question was important enough to the survey that we needed to ask it at all. Or could we have asked something else that would have given us information on the children's socioeconomic status? Or could we simply record silently whether the child was wearing shoes at the time of the interview without asking about it?" R esearcher, Zimbabwe

\section{D2 Has the possibility of community stigma from participation been anticipated and safeguarded against?}

\section{A void stigma by holding community sensitization meetings and using community terminology.}

Singling out selected children and adolescents from a community or classroom for special attention can mark them as different. 

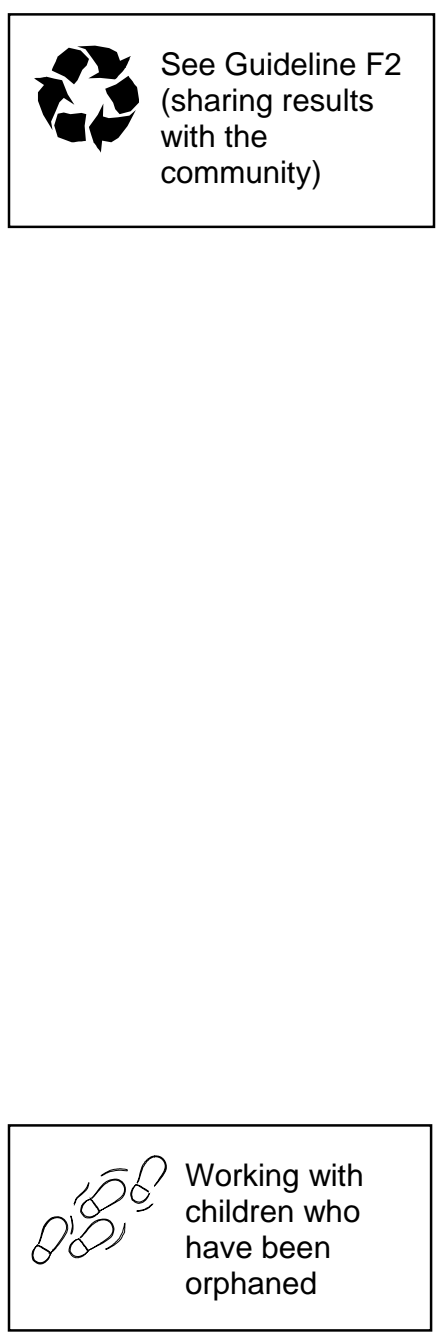

Investigators must make every effort to protect children and adolescents from the unintended consequence of stigma that might be associated with the information-gathering activity.

Holding a community sensitization meeting to raise awareness provides a good opportunity for investigators to try to make sure that community members understand the purpose and limits of the activities, and how and why certain children have been selected to participate in the activity. For example, when working with vulnerable children it is usually not acceptable to distinguish between those who are vulnerable due to HIV / AIDS and those whose vulnerability is due to other causes. There is a great need to work with children and communities to understand how they recognize vulnerability and which groups of children are vulnerable, without introducing or reinforcing stigma.

Investigators must also be sensitive to the language they use to describe the children and adolescents with whom they are working. The use of epidemiologically accurate terms to describe core target groups is often inappropriate when working with real people and communities. Local community groups, children, and staff should be consulted to determine the most appropriate terminology for local use. Instead of using jargon and labels that may stigmatize, investigators should work with local children and adults to find ways to describe the factual characteristics of the groups and situations they are trying to address, such as children who are disabled, children who have been forced into sex work, and so on.

"There are so many orphaned children here in Zimbabwe, even the schools have special rosters of those without one or both parents. O $\mathrm{n}$ one school visit to speak with vulnerable children, the teacher tried to help us by announcing to the class: 'I want all orphans to go outside.' W e were at quite a loss about how to react to this, because publicly identifying orphans can expose them to stigma.

"O ur field officer, who is an orphan himself, was well aware of the problem. $\mathrm{H}$ e tried to explain to the teacher why this announcement was inappropriate. He also suggested that before our team began any future activities, we should hold community meetings in each area, where school heads, community leaders, teachers, and others could find out more about the project, and we could openly address issues of sensitivity, stigmatization, and ethics, and seek agreement on how we can all work together to assist vulnerable children.

"But it's not always simply being identified as an orphan that has a negative connotation. Sometimes we have seen that other children get jealous because an N GO gave new school uniforms or food to the orphans, or simply spent 
time interviewing them. O ther children also wanted the opportunity for oneon-one attention with a caring adult to talk together about their situation.

"To avoid stigma or jealousy, it might be better to interview a wider sample of children, from a variety of backgrounds. Then, during the analysis, attention can be focused on a select group based on demographic characteristics, such as orphans. This method could provide more information about different causes and effects of vulnerability among children. Conducting more interviews would make data collection longer and more expensive, but it would be useful in areas where stigma is a major issue. We have to decide what is most important." Researcher, Zimbabwe

"I feel that the label of orphan should be avoided at all costs within community activities. The use of this term is harmful to community development initiatives. It has a negative affect on the family structure and the development of children's self-reliance. The term 'orphan' does not even exist in many local A frican languages, where children quite naturally refer to

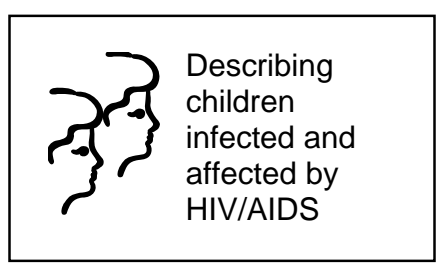
an aunt and uncle as their own parents. The phrase 'children affected by HIV/AIDS' is a much more useful term, with less implied stigma. We must all be mindful that these children affected by HIV/AIDS, however, are not merely statistics to be recorded. Their voices in this debate are vital. Let them have a say in decisions that, after all, affect them." Researcher, Zambia

"W hen our team members visited communities to review our work with vulnerable children, many times they found that community members and health workers openly referred to children as 'A IDS orphans' or used the acronyms CABA (children affected by AIDS) or OVC (orphans and vulnerable children). I even heard children referring to themselves in similar ways, usually with the connotation that to be 'a CABA' or 'an OVC' meant that they were dependent, inferior, or damaged in some way and required additional help or benefits.

"By providing only these children with special services, we marginalize them. They lose the opportunity to help the whole community raise its standard of living. Labeling the children with special terms, which the community then adopts, further intensifies the stigma and the child's sense of being different and alien ated.

"I believe that using these terms robs children of their humanity, when they have al ready suffered. We should simply call them children and describe the characteristics we are seeking to address, such as children who are hungry, children who have a parent that is ill, children who have lost their parents. W e need to hold workshops where children can speak about how they feel on these issues. We must emphasize the need to integrate these children into the community through sports, church, and school-related activities. We must 
find ways to prevent them from being identified as a distinct group." Program manager, Zimbabwe

"Just call me a child. N ot a child with HIV or a child with AIDS. I am just a child. I am no different from other children. I play, work, and do everything just like other children. When I get sick, I visit a doctor... . Then I get better... . I' $m$ not living with a deadly disease as people like to think, but rather living in a deadly world with people who don't understand and are unwelcoming." 13-year-old girl, Thailand²

\section{D3 Can the identities of participants be protected?}

\section{Do not proceed if the safety and security of children and adolescents cannot be assured.}

Investigators working with children and adolescents have a responsibility to keep their identities private. Procedures must be in place to protect children and adolescents from harmful consequences that they might not be able to anticipate if their identity were known. Investigators must consider whether information obtained from children and adolescents will have safety implications for the young people themselves, or for other family or household members. This is especially crucial for the welfare and personal security of participants and their families in situations of political unrest or when working with stigmatized groups.

The principles of anonymity and confidentiality are intended to shield individual participants from negative consequences that result from their participation.

A nonymity: the identity of the individual participant is not collected and cannot be traced from the information provided.

Confidentiality: individual identifiers are collected but identifying information revealed by an individual participant in a relationship of trust is not disclosed to others without permission from the participant. 
Preserving participant anonymity requires that the identity of participants cannot be traced from their records because identifying details are not collected. Anonymous data collection is appropriate when there is no reason to return to the respondents for follow-up, when discussing issues with potential legal and political implications, or when examining a condition as highly stigmatized as HIV/ AIDS.

Alternatives to fully anonymous data collection include linking numerical identifiers to names in a confidential master list that is kept secure, and making sure that all data handling, such as laboratory testing, data entry, and analysis, uses anonymous numerical codes. Participants may also be offered the alternative of a written consent form with a witnessed oral consent that can be signed by an independent witness so they do not have to sign their own name.

Sometimes, for compelling practical reasons, it is not possible to gather information anonymously. For example, a study may require investigators to record children's names or identifiers so they can return to them at a later date to conduct follow-up surveys and/or intervention-related education, care, and support. Under such circumstances, the information provided by participants should be regarded as confidential. It may not be disclosed to others unless under exceptional circumstances. In all cases, investigators should seek to minimize the stigma that may be associated with participation because the highly visible presence of outsiders gathering information in a community makes it difficult to protect the identity of individuals who choose to participate.

Appropriate routine practices that support confidentiality include:

- Keeping named records separate from all other detailed records coded with a study number.

- Training staff to understand the critical importance of confidentiality.

- Limiting access to identifiable data to certain staff members.

- Developing clear guidelines and procedures for collecting, storing, using, and destroying study-related materials.

- Substituting numerical codes to encrypt identifiable data, such as blood test results.

- Removing any pages containing personal information such as names and addresses from survey instruments containing data.

- Recording discussions from focus groups without identifying individual participants. 

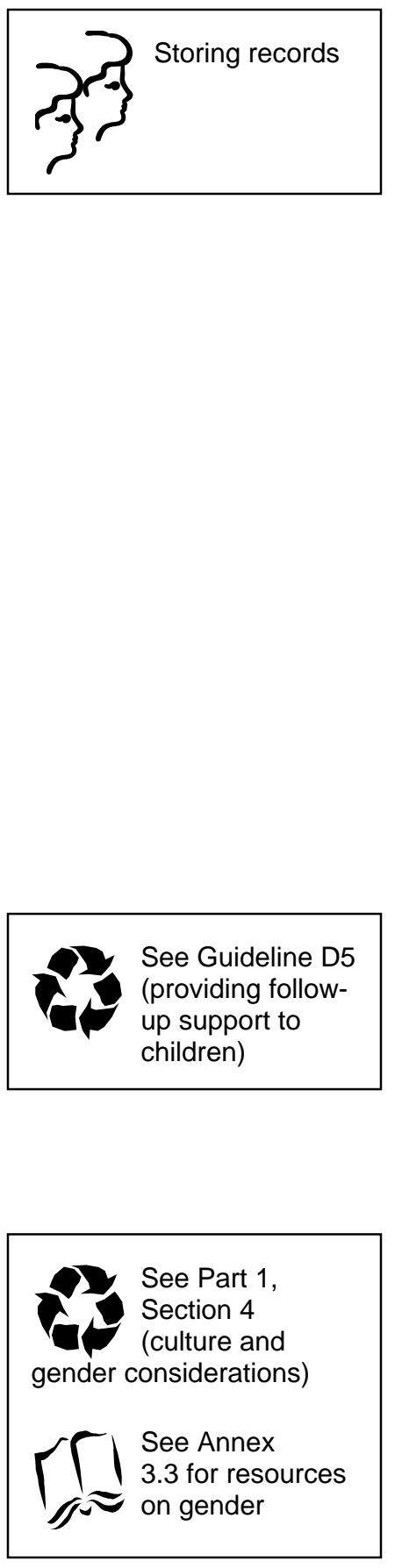

Investigators should make sure that all staff in direct contact with children (including interviewers and their supervisors) have training and experience in working with young people of a similar age. Investigators must train interviewers and supervisors before the activity begins, to ensure that they know how to put children at ease and respond to needs that they might reveal during the interviews, such as psychosocial support.

Interviewer training must also recognize gender concerns, including gender-sensitive interview techniques. Interviewers should be able to recognize when it is appropriate to use only same-sex interviewers. For example, girls may be more likely to reveal abuse to a female interviewer. Interviewer training must recognize the importance of recording the sex of a respondent even when data is collected without personal identifiers.

Once data collection has started, field staff need the support of a chain of supervision. Clear lines of authority and responsibility should be established, to make sure that appropriate staff can respond to the 
needs of children and adolescents as they arise. Investigators should also set up a system to respond to the support needs of the interviewers themselves, who may find their interactions with children emotionally challenging. Appropriate systems may include a peer network to discuss difficult cases or regular opportunities for debriefing with a trained supervisor.

"D uring the interviewer training, we conducted a number of role-plays to get the interviewers thinking about how they would handle various situations. In retrospect, we could have done a lot more on that, but we were not aware of the effect the interviews would have on the interviewers. It was quite a learning experience for us as well. D espite our efforts to train and support the interviewers, most interview ers felt overwhelmed by the responses they received from the children, and helpless to respond. 0 n the positive side, it also galvanized some of them to greater involvement in activities to support vulnerable children through their churches and in their communities. I think supervision is critical to hel ping interviewers themselves cope emotionally with their task. It is al so needed to make sure that interviewers know there is a firm commitment that the information will be used to benefit the people." Researcher, Zambia

"To help them in their role, [the interviewers] must be fully trained in how to provide immediate psychosocial support to the children they are interviewing. They also need to know how to provide support in a safe and culturally acceptable way. For example, because children in Zimbabwe were reporting high levels of abuse, we were careful to instruct interviewers not to touch children. They were not to put an arm around a crying child. They also were told to stay in sight of supervisors when conducting interviews. This was both for the child's comfort and safety, as well as to prevent any action that could be interpreted as inappropriate. H olding a child's hand is probably the most physical contact we would consider permissible.

"It is essential to provide recognition and support to the interview team, including ensuring that they have a reasonable workload, with proper working hours and regular breaks. They also need the reassurance of a functional supervision network that allows them to discuss issues that come up around their work with children. In some cases, the interviewers themselves may have had experiences similar to those of the children they are surveying, such as the loss of loved ones to HIV /AIDS and so on. Short daily debriefings, weekly meetings designed to support the interviewers, and opportunities for individual support may be helpful. It is important that group support time should not turn into a business meeting. It must remain focused on providing emotional support to interviewers. We also gave our interviewers an opportunity to be a part of the preliminary meetings on data interpretation, and asked them to comment on the ethical issues they 
confronted in their fieldwork. In addition to giving the interviewers a chance for closure, this provided useful information for the research team." Researcher, Zimbabwe
See Guideline D6 (responding to serious danger)
A re field staff and community resources prepared to anticipate, recognize, and respond to children's need for follow-up?

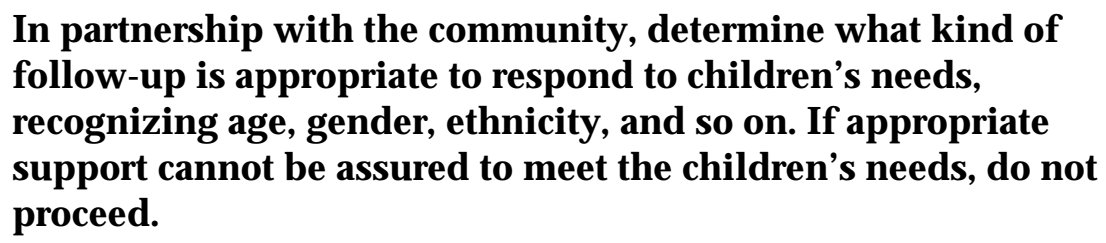

In partnership with the community, determine what kind of follow-up is appropriate to respond to children's needs, recognizing age, gender, ethnicity, and so on. If appropriate support cannot be assured to meet the children's needs, do not proceed.

If interviewers are going to raise difficult topics for discussion with children, investigators must make sure that they can respond to children who are affected by the information they reveal. They must facilitate access to appropriate local resources for follow-up services such as psychosocial counseling or even clinical care, when appropriate. If access to formal services is not possible, another alternative is to bring together groups of young people who can provide each other with peer support. These approaches to follow-up should be explored in discussion with local community members and stakeholders during the initial planning stages. Meetings and individual discussions with community members can help determine what is an appropriate and realistic response to the child or adolescent's needs, recognizing their need for confidentiality.

Often, simply providing information about referral services is not enough, because children and adolescents do not have the resources or independence to contact the appropriate support agencies.

Investigators should try to provide direct access to services when they recognize that the children and adolescents do not have access to transportation or telephone, or when social barriers such as gender, ethnicity, or stigma prevent them from seeking support.

Sometimes agencies that are officially responsible for responding to the child or adolescent's needs are already overloaded and thus unlikely to respond. In such situations, investigators should seek community input to design additional support structures to protect children and adolescents, such as an on-site psychosocial counselor to accompany interviewing teams. They also should consider 
strengthening community resources to cope with increased demand for referrals as a result of the activity.

"O verall, investigators must accept and be comfortable working within the local standard. The reality is that you will not do everything that you want to do for these children. There are constraints related to the availability and accessibility of mental health, child protection, and social services in these countries. R eferral networks may be very difficult to access. For example, we might provide children the number for ChildLine (a child helpline), but how many of the children even have access to a telephone? And how many have the capacity and internal strength to advocate for themselves without help from an adult? O ne way to ensure that you are keeping with the highest standard of care is to have local stakeholders and professionals review the protocols for support and referral, and provide input about what can be realistically done for at-risk children identified by the activity. These are the people who know how the system works and what is truly possible. The best kind of support is to make connections with an existing local group that can either send a psychosocial resource person into the field with the interviewers, or can get someone back to screen at-risk children within 24 hours of the interview. As in clinical settings, one standard I believe we should apply is that an immediate risk of harm is more important than issues of confidentiality. Child safety and protection come first. The way in which reporting and support for the child are handled, however, must be guided by local understandings." Researcher, Zimbabwe

"W e've found that it is essential to have a trained psychosocial support person present to talk with children who become upset during the interview, and to screen at-risk children and provide recommendations for referral. A s a general rule, children you are interviewing need access to immediate support from the field team, on-site screening for more serious problems, and access to referral networks.

"In one study, the interviewers have the opportunity to comment on the child's response to the interview and identify concerns for children in distress. At the end of the interview, children are given a self-administered form to complete in private. The form asks about abuse and whether they would like to receive any further support. Immediately after the interview, the interviewer's comments and the self-administered form both go straight to an on-site supervisor who is responsible for flagging any inconsistencies or further support needs. Children have an opportunity to ask to speak to someone after the interview, and that request is always granted. In addition, the interview er or supervisor may refer the child to the support person, who has experience providing psychosocial care to children. $\mathrm{He}$ or she can assess at-risk children for issues of abuse, suicide, and serious deprivation, such as malnutrition and extreme poverty, as well as serious mental or physical health problems. In some cases, the supervisor is able to provide support on-site. In other 
situations, the individual may talk to the child about a referral for more intense services." Researcher, R wanda
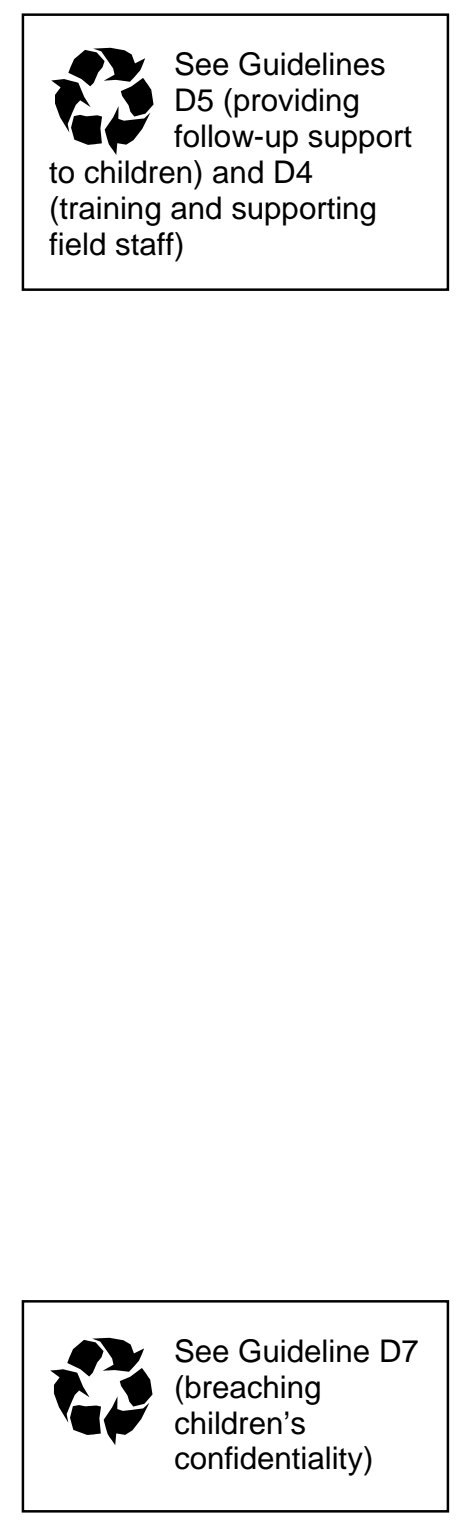

\section{Prepare a reaction plan to anticipate serious needs. If support for the child cannot be assured, do not proceed.}

Investigators' most important responsibility is to protect the child or adolescent from any potential harm that can be anticipated. Before beginning activities, the team must be prepared for the possibility that they will discover dangerous situations, such as abuse or neglect. All staff must be fully trained regarding the local legal issues, regulations, and guidelines surrounding physical and sexual abuse. Training must be gender sensitive to address the specific risk factors faced by girls and boys, and groups that have been marginalized due to socioeconomic or cultural factors, or disability. Investigators must prepare a clear step-by-step plan specifying how the discovery of dangerous situations will be addressed and ensure that all staff coming into direct contact with children are familiar with this plan.

Sometimes groups may want to work with children who are exposed to serious danger and for whom providing protection will be difficult: for example, young people who have been trafficked to work in the sex industry. Under these circumstances, investigators should only initiate an activity if they are prepared to address the dangers of the situation, at least to a standard considered appropriate and acceptable by local community members. Before commencing informationgathering activities, investigators should consult with local stakeholders, including children and adolescents, to understand what constitutes an appropriate response to a dangerous situation and what local groups are al ready available for support. On the basis of this discussion, the investigation team should prepare a timely reaction plan to anticipate possibilities using appropriate local resources. If there are no appropriate local resources available, the investigation team must provide appropriate support mechanisms that address the problem, or otherwise not proceed until such resources are in place.

During the process of seeking informed consent, the interviewer should make sure participants clearly understand that if any form of abuse is discovered, confidentiality will be breached and appropriate actions will be taken to protect the child or adolescent. Before 
beginning the information-gathering activities, investigators must work with the community to establish policies, procedures, and mechanisms to protect participants from danger, using available local resources if abuse is discovered. Young people must be involved in discussions about any concerns, and their views must be taken seriously. Investigators must be sensitive to the consequences for the child revealing the abuse, and whether it is appropriate to discuss the concerns with the parent or caregiver.

The most important concern is to provide protection that meets the child or adolescent's needs and views, recognizing their age, competence, understanding, and ability to effect change. Investigators should consider whether it is necessary to arrange immediately for assistance to provide safety and protection to the child or family members. Investigators must be sensitive to the elevated risks of situations of poverty, conflict, and instability, where there is no guarantee of safety.

"As part of a concerted effort to respond appropriately to children's protection needs, we established a code of conduct and a child protection policy that apply to staff, volunteers, temporary consultants, and researchers. These codes require us to immediately trigger an extensive investigation if the child or young person discloses information indicating they are currently being abused, have been abused in the past, or are at risk of future abuse. H owever, when we started planning a research project interviewing children engaged in sex work to investigate how to meet their needs, we faced significant challenges to the operation of these policies.

"Children engaged in sex work are by definition being abused and exploited. They should be seen as victims of abuse and not as offenders. M any children engaged in commercial sex work come from families with severe problems, including past experiences of abuse. Children, particularly girls, are often forced into prostitution by older men and kept there through the use of drugs and alcohol. Children may also be kept away from their friends and family. Children in such circumstances are especially vulnerable.

"Under these particular circumstances, we had to deviate from normal procedures. We could not alert the authorities to every single instance of child exploitation, otherwise conducting the interviews would have become impossible, and we would have been unable to develop responses to the children's needs at all. But this agreement not to automatically report the child or young person to the statutory authorities must not be interpreted as a blank check in relation to confidentiality. It was still vitally important that all those involved with the activity, especially the children themselves, clearly understood the limits of confidentiality." 4 P rogram manager, Kenya 
"The fact is that in many parts of the world, reporting child abu se does not guarantee a sensitive and positive response from the authorities. This is, of course, a huge problem. But this is not a good enough reason to turn a blind eye to what a child is telling you. While you cannot guarantee a wholly appropriate response from others, it is al so true that researchers by themselves are unlikely to have the skills needed to assist a child who is in an abusive situation. The child will need help from others. The researchers should facilitate this, involving the child as much as possible in any decisions." Researcher, Zimbabwe

\section{D7 Do all staff know the circumstances under which participant confidentiality should be breached?}

\section{Confidentiality should be breached if a child or adolescent requires immediate protection. Staff must make sure that participants are aware of this before asking for any information.}

The duty to provide immediate protection to the child or adolescent is more important than the duty to maintain confidentiality. Although every effort must be made to preserve confidentiality when possible, the following three situations require an interviewer to breach confidentiality and contact an appropriate organization that can provide help and protection to the child:

- Discovering that a child is being abused or neglected.

- Discovering that a child is harming or threatening harm to another person.

- Discovering that a child is harming or threatening harm to him or herself.

The decision to seek outside help for a child in a situation that might be dangerous must be discussed with the child and, when appropriate, the parent or guardian. Local staff familiar with the cultural context should try to assess the following factors when determining whether confidentiality should be breached:

- What are the risks faced by the child?

- Does the situation need immediate attention?

- How likely is it that these reports of abuse are true?

- Is the young person al ready receiving services that recognize this situation? 
- What are the child's expectations of the role of the interviewer?

- What is the likelihood that reporting the situation will actually provide protection to the child? 5

Child participants and their adult guardians consenting to provide confidential information must clearly understand when the promise of confidentiality will be broken and to whom. The interviewer is responsible for taking the time to make sure participants and guardians clearly understand what this means at the start of every interview.

Every situation is unique and must be evaluated independently. Special considerations will apply in every situation. Examples include:

- When a child is clearly not in charge of his or her own safety, such as a child being held against his or her will.

- When other children are also at risk, such as siblings also living in the household.

- When an alleged abuser is in a position of trust, such as a parent, caregiver, teacher, or NGO staff member.

In some circumstances, staff may be legally obligated to report events to a local authority, such as a child welfare department or community leaders. This may be done only after discussion with the child.

\section{E. Are procedures in place to ensure that the activity proceeds ethically?}

This section examines the safeguards that investigators, having decided that the activity is necessary and ethically justified, must put into place to ensure that the activity proceeds in accordance with ethical standards.

\section{E1 Do all investigators know who can give informed consent when working with children and adolescents?}

\section{Children must give their agreement to participate. Consent is also required from appropriate adults.}

Investigators must seek the agreement of children and adolescents and those responsible for them before gathering information from them. 

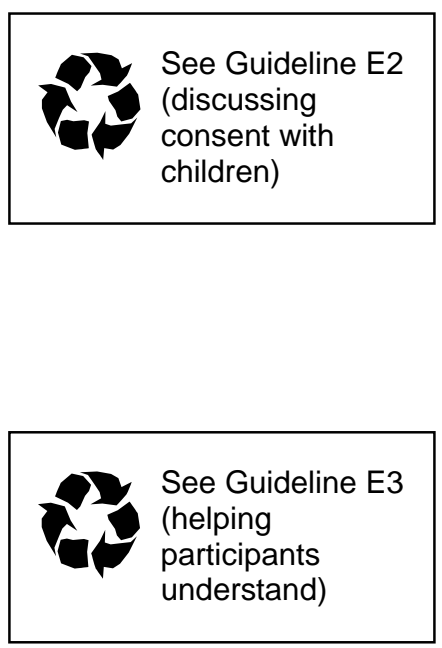

Hidden or covert research, where children or adolescents do not know they are being observed or having their words recorded, is absolutely unacceptable.

A mong adults, agreeing to participate in the activity voluntarily and with understanding is called informed consent. Children's ability to understand what is required of them varies according to their stage of development and the context of the information-gathering activity. Seeking consent from young people under the age of majority requires investigators to obtain and document the following:

1. Active agreement from the child, recognizing the child's age and maturity (i.e. obtaining the child's assent).

The active agreement of the child, or the child's assent, is an absolute ethical requirement. A lack of refusal is not enough. Children who agree to take part in the information-gathering activity must understand that they can stop the interview at any time and refuse to answer any question if they choose. Investigators must be sensitive to the possibility of a parent pressuring a child to agree and look out for non-verbal clues from the child indicating discomfort with the activity. They must verify that children have the opportunity to make their own decisions in an age-appropriate manner.

2. Agreement from the child's parent or guardian, where available (i.e. obtaining parental/ guardian consent).

In most countries, agreement or assent from a child has no legal standing. ${ }^{6}$ For legal purposes, children and adolescents below the age of majority are usually presumed incompetent in the decisionmaking process. The parent or guardian is regarded as the best protector of the interests of the minor. If either the child or the caretaker does not agree, the child should not be included in the activity. Consent is legally required from one parent or guardian, but this process must be conducted with due sensitivity to family dynamics.

Parental consent must be sought according to local practice. In some countries (e.g., the United States), ethical review boards will accept only active parental consent procedures (e.g., if a child takes a written form home from school, their parent/ guardian must actively complete and return it indicating their agreement to the child's participation). In other settings (e.g., South A frica), ethical review boards are aware that active parental consent requirements may result in bias or forgery, and will accept passive parental consent procedures (e.g., if a child takes a letter home from school 
to their parent/ guardian, they are assumed to have given consent unless they object). ${ }^{7}$

3. Agreement from other adults interacting with the child in the study (i.e. obtaining additional consent or permission).

It is sometimes necessary to seek consent from additional adults who interact with the child, such as a teacher, social worker, or health care staff member. Consent from an additional adult is also required if the parent or guardian is unavailable to provide consent, or if it cannot be assured that the parent or guardian will represent the child or adolescent's best interests. In such cases, a child advocate can oversee the assent process to make sure adequate protection is provided to the child or adolescent.

To minimize the potential for harm that might arise from misunderstandings and the desire to conform to community expectations, the seeking of consent, assent, and permission should be conducted privately with each individual.

"We carried out qualitative and quantitative studies on psychosocial issues facing children and adolescents between the ages of 6-12 and 13-18. When we asked for consent from parents/guardians for the interviews, we spelled out the nature of the questions so that they knew we were including questions of a sexual nature for the older children, addressing relationships with family members, including themselves, and other issues. We stressed the importance of confidentiality and that we wanted to minimize the emotional response stirred up by the questions. We provided guardians and the children and adolescents with a referral list of local resources for assistance. We had a very low refusal rate. A t first, some of our staff were hesitant to use such a detailed consent form, feeling that most guardians would be frightened off the project. I think the reality is that participants actually respected the informed consent process and the idea of knowing exactly what they were getting themselves into. They welcomed receiving information about the consequences of their participation - both positive and negative. It also hel ped protect the organizations carrying out the activity." Researcher, Z ambia

"We conducted surveys with young people in three towns in Ghana, to explore knowledge and attitudes about sexual and reproductive health, gender, and drug abuse issues, and to assess their access to services.

"W e encountered youth in a variety of situations, including children looking after younger children, often siblings. If the interviewer was convinced that the children had no adult guardian and their participation was appropriate for the study, then they sought additional consent for the interview from community leaders as well as the children themselves. 
Family consent in Uganda
See Guideline E5 (incentives and avoiding coercion)
"W einterviewed older adolescents in schools or in settings of trade apprenticeships, such as hairdressing, dressmaking, carpentry, and so on. In these cases, in addition to discussions with the students, we also approached the school or trade authorities in advance to ask them to communicate with parents for their consent.

"W e also con ducted a separate survey of youth living on the streets, who were working as hawkers and porters. Where possible, we asked for consent from the leaders of associations who worked with these children. But in most cases, children did not belong to any association. It was especially important that the interviewers explained the purpose of the study to every individual and made sure that they knew they had the right to refuse to participate or answer specific questions if they did not feel comfortable." Program manager, G hana

"A ccording to U gandan law, an 18-year-old male living at home has the legal right to make his own decisions; however, it is customary to gain the consent of his father prior to entering into any obligation or contract, including participation in research. $U$ gandan women may often refuse to make a decision about their child's participation in research without the consent of their partner. Participants at a workshop on family consent suggested the following compromise solution: a waiting period of 48 hours between the time individuals were approached about participation and the time they signed the consent form. This gave them an opportunity to consult with family members if they chose to before making the final decision about participation. The group recommendation for delaying consent accommodates cultural norms regarding family involvement in decision-making without lessening the importance of respect for the individual." 8 R esearcher, U ganda

\section{E2 Do interviewers know the important elements of consent to discuss with all children?}

\section{Interviewers must make sure that children know they can stop or withdraw at any time.}

Before the start of an interview, interviewers must explain to children and adolescents that they have the right to make their own decision about taking part in the activity:

- they can have time without pressure to think about their decision;

- they can ask the investigator questions about their decision;

- they can discuss their decision with a friend; 
- they can refuse to participate from the beginning;

- they can refuse to answer any question; and

- they can stop the interview at any time.

The interviewer should make sure that the child or adolescent knows that they do not have to give a reason for refusing to take part, and that refusal and withdrawal will not have any negative consequences.

Interviewers must explain their role clearly to the young participants, invite active questioning, and establish that the children are free to say what they want. Investigators should be responsive to any nonverbal indications that show a desire to discontinue, especially from young children.

Power and gender dynamics between children and adolescents and the investigator may make it difficult for young participants to indicate a desire to withdraw from the activity. One way of empowering young respondents to be more in control of the interview is for the interviewer to establish and practice with them a signal they can use at any time if they want to stop. The interviewer should also make sure that children know they can still stay with them for a time period equivalent to the normal interview time if leaving early would publicly reveal their decision to withdraw.

The interviewer should provide information about the activity to children and adolescents of all ages, in an age-appropriate way. Discussions with children in the community and field-testing of the consent process are necessary to determine what information is appropriate and can be understood. It is often helpful to provide this information in written form, so that it can be considered and discussed later.

\section{E3}

Have investigators ensured that child participants and adult guardians fully understand the nature of the activity?

Provide children and adolescents and their parents or guardians with information about the activity in a manner appropriate to their culture and education. Consent forms and informational tools should be developed with community members and fieldtested.
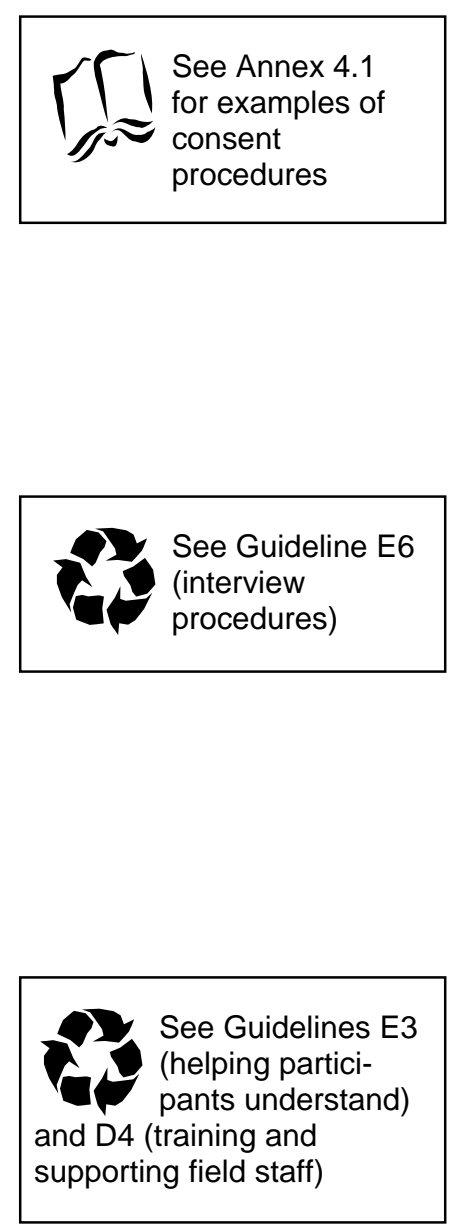
See Guideline E2

(discussing

consent with children)
See Guideline D7

(breaching

children's confidentiality)
After making sure that participants and guardians understand that they have the right to refuse or withdraw at any time, providing more information about the activity is essential. Deciding how much information to provide without overwhelming, confusing, or pressuring the participants can be a difficult balance. This is a decision that should be made in close consultation with a community advisory board, and may be examined closely by an ethical review board.

At a minimum, investigators should explain9:

- the purpose of the activity;

- the procedures involved in the activity, such as interviews or focus group discussions;

- $\quad$ any risks and benefits the participant can reasonably expect as an individual or for the community; and

- the circumstances under which the confidentiality of the information about individual participants will be breached.

Additional helpful information includes:

- the expected duration of the activity;

- the investigator's responsibility, if any, to provide follow-up;

- the name and contact details of a person to contact with any concerns;

- the conditions under which the investigator may ask the participant to leave the activity;

- any potential conflicts of interest, including who is funding the activity;

- how any written records and biological specimens will be stored and used;

- how results will be communicated to participants and other audiences;

- other sources of information about the activity; and

- whether an intervention that has been demonstrated to be effective will be made available.

Forms and supporting information (such as leaflets) should be designed to reflect the needs of the local community. They must be developed in close consultation with community representatives, including field-testing. Where appropriate, investigators should anticipate the needs of adults and young people with little formal education. For example, in some situations it might be necessary to make sure the consent or assent form can be read aloud in 
comprehensible language, and that a written symbol rather than a signature can indicate consent. Community meetings are often a helpful way to provide support and information about this process, and they also provide an opportunity for discussion.

"During community meetings, the concept of randomization was explained successfully by using agricultural terms involving different seed varieties that were familiar to farmers in the area. Communicating information about a choice and its implications can be difficult and time-consuming, but it allows valid, informed decisions. We found that widespread illiteracy is not a barrier to comprehension, especially since informed consent is more an interactive process than one that depends on reading." 10 R esearcher, Senegal

E4

H as the use of a child advocate to represent the best interests of the child been considered?

\section{Use an independent advocate to represent the views of children if there is any doubt about the protection provided by their guardians.}

Sometimes children do not have an effective guardian who can represent their best interests (for example, children living on the street who are not in contact with their families, or children living in childheaded households following the death of their parents). Sometimes there is serious doubt about whether the guardian's interests adequately reflect the interests of the child, for example in the cases of children at risk of abuse within the home.

In such cases, investigators should appoint an independent advocate who can represent the interests of the child or adolescent. The advocate is responsible for providing informed consent and for upholding protection from harm throughout the activity.

Permission to seek consent through a child advocate instead of a guardian may be required by the ethical review board. Procedures must be determined in consultation with community representatives and consistent with local law and practice. A child advocate may act on behalf of a child whose parent is sick or unavailable, but if the parent later becomes able to provide informed consent, their decision must be sought and respected.

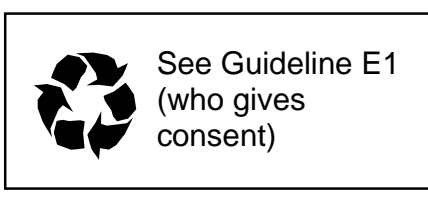

Explaining complicated concepts with local ideas 
See Guideline E1 (who gives consent)
Child advocates
The child advocate must be familiar with the child's circumstances and be independent of any other interests, including the informationgathering activity. For example, an investigator working with children in institutional care may require additional consent from the institution's director, but an independent advocate is also required to ensure that the child or adolescent's interests are protected. Depending on the nature of the information-gathering activities, a child advocate may act on behalf of an individual child or adolescent or may represent the interests of a broader group of individual young people within a single activity.

For example, guidelines for the appointment of a child advocate for research funded by the United States are specified under law, and provide an example that is also useful for programmatic activities:

"The advocate shall be an individual who has the background and experience to act in, and agrees to act in, the best interests of the child for the duration of the child's participation in the research and who is not associated in any way (except in the role as advocate or member of the Institutional Review Board) with the research, the investigator(s), or the guardian organization." ${ }^{11}$

\section{The independent advocate is responsible for ${ }^{12}$ :}

- verifying a minor's understanding of the assent procedures;

- supporting the minor's preferences;

- ensuring that participation is voluntary;

- monitoring reactions; and

- ensuring adequate follow-up.

"W e were conducting an assessment in Zambia of the needs of orphans, vulnerable children, and their guardians. O ur teams worked in pairs to collect data from children aged between 6 and 15. O ne person, trained in how to communicate sensitively with children, was responsible for conducting the interview, while the other was a trained social worker or counsel or who provided psychosocial support and acted as an advocate to represent the child's best interests. Both team members had previously worked with children and families through local N GO s in the same districts. They were trained on the psychosocial issues faced by orphans and vulnerable children, and how to protect the child's best inter ests while completing the questionnaire. The training covered issues of parental death and illness, fears and anxieties around being relocated, emotional trauma of being separated from siblings, loss of family home and possessions, and cognitive capacity to comprehend what had happened. 
"The psychosocial support person was present to ensure that the child understood the survey, and to protect their best interests, especially if the interaction became emotionally difficult for the child. In a few cases, the psychosocial support person had to intervene and stop interviews because it was just too difficult for the child to talk about his or her deceased parents. In these cases, the psychosocial support person provided individual on-the-spot counseling, and offered referrals to a local psychosocial support organization that had been previously contacted about the survey." Researcher, Z ambia

"W e conducted interviews with street children in Cambodia to develop a better understanding of their vulnerability to HIV, in order to design appropriate intervention activities. The children, who were aged 11-18, were recruited from a drop-in center run by a local N GO. Parental consent could not be obtained because parents were either unavailable or would not act in the best interests of the children.

"We recruited a team of child advocates whose role was to protect the children's interests and needs throughout the study recruitment and interview process. The independent team of child advocates included representatives of ministries, legal organizations, child rights groups, and NGOs working with street children. M ale and female advocates were available to represent children of the same sex. Child advocates were un paid. They received training and supervision regarding child protection issues, confidentiality, and research ethics from U NICEF and the local NGO.

"A child advocate was present in the room with the child during the entire recruitment and interview process. The advocate could interrupt, overrule, or terminate the process at any stage if he or she felt that the child was feeling uncomfortable or pressured. The child advocate could al so assist in the explanation of the study to the child or rew ord questions if necessary. In some cases, when children appeared scared to speak about their involvement in illegal activities such as theft, the child advocate was able to find the right words to increase the child's confidence in the confidentiality of the research.

"The child advocate sat at a distance from the child but within the child's sight, while the interviewer sat in front of the child. The presence of the child advocate in the room did not generally appear to make the child feel uncomfortable. In general, the children appreciated the efforts that were made to respect them as individuals. They appreciated the fact that higher-class adults, with whom they would otherwise have no relationship, sat down on the ground with them, shared tea and bananas, and listened to them.

"Some children misunderstood the role of the advocate and had very high expectations that they could provide them with continued help after the interview. O nce, a small group of older boys asked for direct protection from one of the advocates against a gang. They were disappointed to learn that legal action was a long process involving police who they said backed the gang. The boys concluded that 'it is the same old stuff' and 'nothing ever changes.' 
Following this incident, the research team held a meeting with the advocates to clarify their role. Their monitoring was limited to the research period but they could, on their own initiative and independently, offer legal services to the children. A dditional follow-up services were available through the local NGO drop-in center, if required.

Further work must be done to assess how their presence benefited the children during the interviews and make sure that children understand the role of the child advocates." Program manager, Cambodia

\section{E5 Have procedures been put in place to avoid undue influence or coercion?}

\section{A void efforts to unduly influence participation by the use of incentives. If incentives are used, they must be in line with local living standards.}

Incentives have been used with adults to compensate participants for their time, travel, and inconvenience. To protect children and adolescents from inappropriate pressure, the use of incentives must be handled carefully. Children and families living in situations of extreme poverty are especially vulnerable to coercion, exploitation, and bribery. Incentives must not be used to relieve responsibility if there is the risk of harm to children and adolescents.

Incentives must be used sparingly to avoid raising children's expectations and causing later disappointment. Investigators must be sensitive to the possibility of pressure from children's families or peers to get the incentive. If used, incentives must be in line with local living standards to avoid unduly influencing an individual's decision to participate.

Community incentives may be given to schools or trusted community leaders rather than individual participants to avoid pressure on individuals (for example, school supplies). Community incentives may be used for the benefit of the children involved in the activity, their families, and their community members. This should be discussed with broad representation of community members and stakeholders to ensure fair distribution and avoid the undue influence of community members with commercial, political, or other special interests.

If it is necessary to provide incentives to individuals, these must be handled sensitively and in consultation with community members. If 
used, they should belinked to the children's efforts to attend an interview or meeting. In order to avoid pressuring children, incentives must be independent of their responses and behavior during the interaction, including the decision to end theinterview early, or not to answer certain questions. It is appropriate to compensate individual participants for the time and effort that they have put into the activity, for example by providing local refreshments and refunds for travel. However, such compensation should not be used as an incentive to influence participation.

"W e are conducting a research study to explore ways to provide psychosocial support to orphans and vulnerable children in Zimbabwe. O ur ethical review boards in the U nited States and Z imbabwe set guidelines that prevented us from offering children any money or food as a reward for doing an interview with us. We found this a difficult approach to take because of the current economic crisis in Zimbabwe and the severe financial hardships facing many families today. We even had trouble with rumors that were spread by groups of young people who would advise other youth not to show up for the interview because they weren't going to receive anything.

"In a couple of areas children walked eight kilometers or more for an interview, and often made the journey on an empty stomach. D ue to the levels of extreme need we encountered in rural areas, just before the children were due to head home we announced without forewarning that we were offering simple lunches or snacks to the children as a gesture of goodwill. Respondents and interviewers were able to sit together and enjoy a simple plate of rice and soup.

"A nother thing we were able to do was to go back to each community's Gogo (female elder) after the interviews and offer her a token of appreciation. We would give each Gogo bags of sugar beans and high-protein biscuits that she could keep for herself or distribute to the children in her community as she saw necessary." Researcher, Zimbabwe

"The kind of incentives we use depends on the group being considered. We al ways try to make sure that the young people understand that the incentive is not a payment; rather it is to be used for refreshment when they are participating in our activities. Sometimes participants would prefer to receive a little money rather than refreshment because they might consider something like a soda to be wasteful. We try to avoid giving young people too much money for their participation, because it would definitely have a negative impact on their participation in other projects in their communities when money is not available. Also, sometimes we do not inform them that they will receive incentives before they participate because we want them to decide to participate of their own free will and not because of any form of incentive. 
"Some incentives we have used for our work with young people include local refreshments, a token amount of money, local candies (for young children), lunch (if participation takes a lot of time), and school supplies, such as pens, pencils, and notepads." Program manager, Ghana

See Part 1, Section 3 (legal and professional requirements) for more information on ethical supervision
See Part 1,

Section 4

(culture and gender considerations)
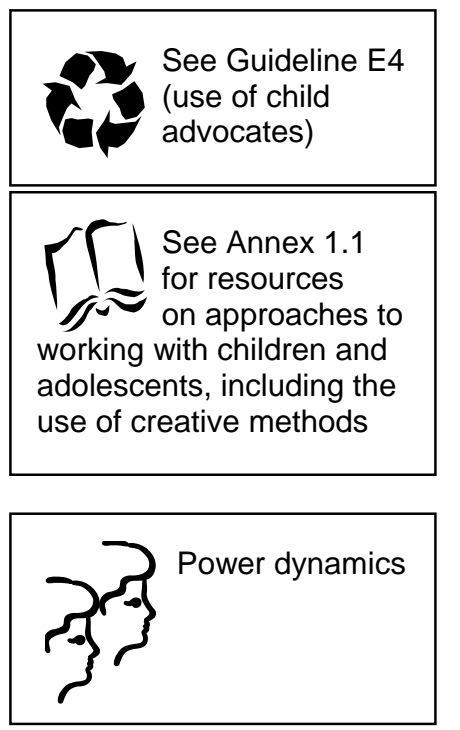

\section{E6}

\section{A re children's needs for support and protection reflected in the interview procedures?}

\section{Interview procedures should reflect the need to protect the best interests of the children and adolescents. Consult with community members to determine appropriate practices.}

To protect the child, it is best to hold interviews in a visible place where they cannot be overheard. Some interviews may require privacy to hide the involvement of the child. Investigators should work in partnership with child caretakers and communities to determine appropriate practices.

Investigators should consult with community members and local child welfare staff to ensure that cultural and developmental standards for children and adolescents in this setting are followed, including how the sex of the interviewer and interviewee will affect practices. For example, does the community frown on a young girl being alone with an older male outsider?

Interviewers must recognize that the children they are working with are vulnerable because of the difference in power created by two factors: the age difference and the difference in the social standings between the participant and the interviewer. The additional presence of a caregiver or a child advocate to monitor protection of the child's interests should be considered if the child is particularly young. If a child requests the presence of an adult or friend for reassurance, this should be respected. Information from interviews conducted in the presence of an additional adult must be identifiable in case this causes bias that affects results when they are interpreted.

"It is sometimes hel pful to define the role of researchers in a way that reduces social distance between them and the children. 0 ne helpful way is to define the role as that of students who want to learn from their experience. It may al so be hel pful to emphasize one's own ignorance or incompetence. It can be empowering for children to know that they have information that researchers do not. It may also be helpful for researchers to share with children a little 
about themselves, so that the children are able to see them as whole persons rather than as powerful adults that they don't know." ${ }^{13}$ Researcher, UK

"I found a big problem with interviewing at the village level was that everyone wanted to listen in, or at least to hang around to see what the stranger was doing. It is at times difficult to tell these people (usually children) to move back. W ith the neighbors listening, it is difficult for interviewees to express their own opinions and beliefs. They tend to givea community-appropriate response instead. Interviewing outside in a visible place, such as under a tree, is sometimes preferable to interviewing inside where no one can see what is happening. At least outside people can watch what is happening, and you can set perimeters so people are far enough away that they can't hear the questions and answers." Researcher

\section{F. Is there a clear plan and adequate funding for follow- up activities?}

This section examines the responsibilities of investigators after the information-gathering process is complete, as the findings are released and used.

\section{F1 Is there a clear plan and adequate funding for secure storage of written records when the activity is over?}

\section{Use appropriate procedures to maintain the safety and security of participants.}

Investigators are responsible for protecting children and adolescents throughout the activity and beyond. This includes keeping their confidentiality and anonymity when findings are disclosed and storing or destroying written records. Investigators must be aware of the social and political implications of their findings for participants. They are responsible for keeping written records secure for as long as they are needed, or else destroying them. If records are kept, they must make sure that there is enough money available to keep up confidentiality in the storage. 


\section{F2 Is there a clear plan and adequate funding to give community members and partner organizations access to the results of the activity?}

\section{Share findings with community members in an accessible, appropriate format.}

Before the activity begins, the team must develop and agree on a plan to release the future results to community members and all stakeholders and partners. The plan must recognize that different groups have different needs for information (for example, community members, local political leaders) and include an adequate budget to meet agreed needs.

One way that information-gathering activities can benefit children and adolescents is to share the results with them and discuss their reactions. Investigators also have an ethical obligation to share their findings with the individuals and communities who helped them gather the information. They should do this as soon as the activity is finished, in a way that makes sense to the children and their guardians, taking into account their age and level of education. Holding a meeting to discuss findings with participants and their parents or guardians may have the additional benefit of helping investigators understand how the participants interpret the results. If any information is withheld, it must not result in harm to the participants.

Investigators must be careful to safeguard participants' identities when reporting results, especially to community members. They should think carefully about the effect their words might have on a vulnerable family, particularly how the child might interpret what is said. Investigators must work with local informants and communications staff to make sure that participants' real names and any other identifying details are not revealed. This consideration is especially important when information is provided under politically unstable circumstances, or when the information appears in connection with a highly stigmatized condition such as HIV/ AIDS.

Strategies for releasing information should recognize the different needs of local community groups, children's service providers, national or international press activities, or research publications, among others. Community members and participants should bekept informed about who will be receiving this information and how it will be used. 
If photographs of the participants are used to illustrate publications, the investigator and photographer must confirm that the photographs are not a threat to anyone's safety or confidentiality. They must consider carefully who will have access to the photographs, the negatives, and the publication. All photos must reflect the true situation. The individual must consent to the use of the photograph and the photograph must not be misleading. Images that do not reveal a child's identity should be considered, such as a silhouette, profile, cropped image of part of a face, or a blurred or darkened image. If photographs are staged or models are used, this must be clearly stated.

"It's important to let the community members and leaders know that we don't intend to just gather information about their children and then forget about them. At the initial community meetings when we introduce the project, we make it clear that we are interested in working with the community even after all the data has been collected. We explain that we will return before we release the data to ask for their help interpreting the findings, and we talk about how these results will be used." Researcher

"W e conducted a training course with young people in two provinces of northern Zambia. To examine the impact of the training, we conducted surveys and focus groups before and after the course, and compared results. W e went back to the schools and the youth clubs where we had originally met the participants and held meetings to talk about what we had found. Team members (who were originally our interviewers) talked with the young people about the findings, using a structured facilitation guide.

"First, they confirmed that participants were familiar with the use of percentages, and explained that they were going to present group results without any individual information. Then they described the main findings in simple language, and displayed them on large sheets of paper pinned to classroom walls. A II participants received a leaflet written in very clear and simple language that explained what we had done, described the findings, and suggested some ideas for group discussion. W e encouraged participants to share and discuss the information with their friends and families. We prepared two different versions of the leaflet, one for each province, so that participants would receive the results most relevant to their area. The leaflets also included a message of thanks and provided contact details for any questions.

"At the meetings, participants had the opportunity to ask our team any questions they wanted. They told us that they appreciated the opportunity to find out what the surveys had shown, and how their information would be used. The discussions with the young people shed light on some really unexpected results. As researchers, it gave us a really valuable opportunity to confirm that what we had found was credible." R esearcher, Zambia
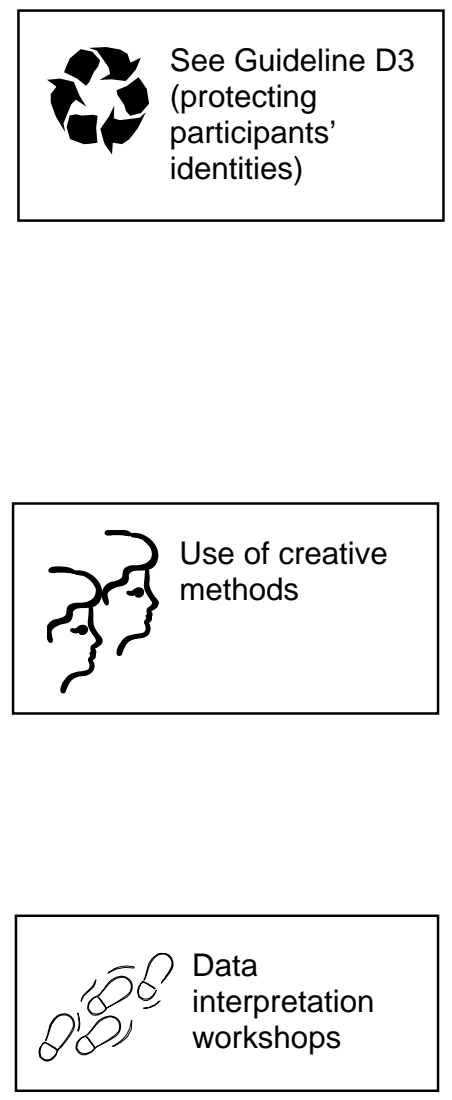


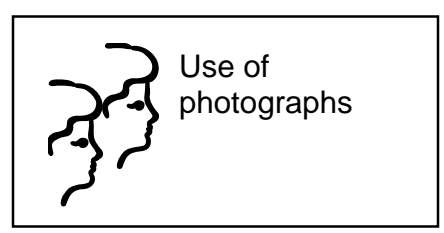

"W hen I get out a camera to take photographs of the children we've been working with, it provokes an extremely enthusiastic response. They all want to be in the picture. But I wonder, are they aware how their photo might be used? What would they think if they knew that people overseas would see their picture in a publication from an organization associated with HIV and AIDS? A young adolescent who willingly identifies himself as an orphan who lost his parents to AIDS may regret that revelation and the resulting consequences as a young adult, when it comes to marriage or job opportunities. I think that the use of photographs under these circumstances is seldom appropriate and should be limited because appropriate written consent regarding long-term consequences is rarely possible or meaningful." Program manager

${ }^{1}$ Seek advice from a statistician regarding how to calculate sample size, including considerations of statistical "power." Many statistics websites provide online calculators.

2 Medecins Sans Frontières, http:/ / aids2004.msf.org.hk/ en/ PaintingEx.htm.

${ }^{3}$ Quoted in “Human research must protect participants," FHI N etwork. 2002. 21(2).

${ }^{4}$ Joseph Rowntree Foundation and Save the Children UK.

${ }^{5}$ A dapted from Fisher, C.B. et al. 2002. "Research ethics for mental health science involving ethnic minority children and youths," A merican Psychology 57(12):1024-40.

${ }^{6} \mathrm{~A}$ notable exception is the United Kingdom, where the principle of "Gillick competence" provides the opportunity for even very young children to give consent under certain circumstances. For a fuller discussion, see Alderson, P. and V. Morrow. 2004. Ethics, Social Research and Consulting with Children and Y oung People. London: Barnardo's. http:/ / www.barnardos.org.uk/ shop/ publications/ acatalog/ Publications_Shop_Society_124. html.

7 Mathews, C. et al. 2005. “Written parental consent in school-based HIV/ AIDS prevention research," A merican Journal of Public H ealth 95(7):1266-69.

8 Sana Loue, David Okello, and Medi Kawuma. 1996. "Research bioethics in the Ugandan context: A program summary," Journal of Law, M edicine \& Ethics, 24(1):47-53.

${ }^{9}$ Based on Wood, S. Y., B. A. Friedland, and C. E. M cGrory. 2002. “Informed consent: From good intentions to sound practices," Population Council-Ebert Program Publication Series. New York: Population Council. http:/ / www.popcouncil.org/ pdfs/ ebert/ informedconsent.pdf.

10 Preziosi, M., A. Yam, and M. N diaye. 1997. "Practical experiences in obtaining informed consent for a vaccine trial in rural Africa." N ew England Journal of M edicine 336(5):370-73.

${ }^{11}$ Code of Federal Regulations: Title 45; Public Welfare Department of Health and Human Services: National Institutes of Health/ Office For Protection From Research, http:/ / ohrp.osophs.dhhs.gov/ humansubjects/ guidance/ 45cfr46.htm\#.

12 Hoagwood, K. et al. "Ethical issues in mental health research with children and adolescents" quoted in Fisher, C. B. et al. 2002. "Research ethics for mental health science involving ethnic minority children and youths," A merican Psychology 57(12):1024-40.

13 Mann, Gillian and David Tolfree. 2003. "Children's participation in research: Reflections from the care and protection of separated children in Emergencies Project." Save the Children Sweden: Stockholm. 


\section{Part 3 - Summary and Recommendations}

This publication is intended to promote ethical practices among investigators who gather information from children and adolescents to develop services meeting their needs. The primary concern in all such activities must al ways be the protection of the best interests of the children and adolescents. All of the guidelines in this document suggest ways in which to put this value into practice.

\section{These guidelines are intended to safeguard children and adolescents, and following them requires special protection measures to be put into place. Activities should not begin unless investigators have secured an adequate budget to address the consequences of these needs. M onitoring adherence to the ethical standards recommended by this publication must continue as an ongoing process throughout the duration of the activity.}

The recommendations in this document present suggestions to be applied under ideal situations of adequate funding and human resources. Implementing the recommendations in this publication will require cooperation and support among all individuals and organizations collaborating on information-gathering activities. Although attention to these standards is especially important in the early stages of planning the activity, they apply at all stages throughout its duration to all people involved. Participants must be protected from the beginning of their involvement through to the release of findings, and even beyond completion of the activity, with the responsible storage of records. Absolute requirements for a minimum package of responses are:

- providing children and guardians with the opportunity to give informed consent to their involvement in the activity;

- consultation with community members regarding local acceptability of the activity; and

- existence of functional referral systems to respond to the circumstances revealed by the activity.

Beyond these absolute requirements, the key recommendations underlying all the guidel ines include the following:

1. Balance the need to maximize children's participation by finding out about their own opinions on the issues affecting their lives with the need to minimize their exposure to harm. 
2. Careful advance planning is crucial. Investigators are responsible for thinking through all possible consequences, both intentional and unintentional, of the information-gathering activity and for anticipating the effect of the activity on the young people and their families. If appropriate safeguards cannot be put into place, the activity should not proceed.

3. Discussions should be held with local community members, including children and adolescents, whenever possible. Community meetings at different stages of the informationgathering activity can servea variety of purposes, including sensitization, review, and interpretation. These discussions can serve the dual purpose of improving adherence to ethical standards and improving the quality of the information gathered.

4. If there is any question about whether data collection could be harmful to children, only begin the activity if services are in place to address possible consequences. If the information-gathering activity is not associated with a service, prepare referral information for children to get the required support. Do not conduct the activity without functional support systems in place.

Staff members working with children and adolescents are responsible for upholding these ethical standards according to their different roles and responsibilities. Recommended roles and responsibilities for these different individuals and groups are outlined in the table on page 60.

\section{Additional considerations}

\section{Sanctions for non-compliance}

While this publication is intended to address concerns expressed by responsible staff al ready struggling with these issues, compliance with the recommendations remains voluntary. Investigators tempted to overlook any of these ethical considerations should remember that although compliance with these recommendations may be unenforceable, many possible sanctions exist for non-compliance. For example:

- the activity may be terminated by sponsoring organization;

- any products related to the activity may be embargoed;

- the investigator or sponsoring institution may be disqualified from further funding; and

- the investigator may be barred from futureactivities; or 
- the investigator or sponsoring organization may be personally subject to a civil lawsuit.

\section{Scale of the activity}

M ost of the case studies described within this document describe activities taking place within a single project site or community. Although the same basic principles apply when considering activities with a broader regional or national scope, practical considerations may require functionally different applications of these principles. For example, a community advisory board overseeing a large-scal e activity should include regional representatives acting on behalf of diverse communities in their areas. Dissemination mechanisms for a nationalscale activity should make use of multiple methods of feedback, including local newspapers and stakeholder groups, as well as local community meetings. Regional representatives may be invited to a central dissemination workshop at which they are trained and provided with dissemination materials in order to conduct local dissemination workshops within their community. 


\section{Recommended roles and responsibilities of key actors}

\begin{tabular}{|c|c|}
\hline Key Actors & Roles and Responsibilities \\
\hline $\begin{array}{l}\text { Organizations } \\
\text { providing } \\
\text { funding to } \\
\text { support } \\
\text { activities with } \\
\text { children, } \\
\text { including donor } \\
\text { agencies and } \\
\text { implementers }\end{array}$ & $\begin{array}{l}\text { - Establish and support review policies and procedures (e.g., Community Advisory Board and } \\
\text { Ethical Review Board) to approve new activities and oversee ongoing activities to safeguard } \\
\text { the protection of participants. } \\
\text { - Ensure that the review mechanism is equipped to respond to the specific needs of children } \\
\text { and adolescents by including the views of community members and qualified professionals } \\
\text { and providing them with the necessary funding to function. } \\
\text { - Define policies and procedures for promoting integrity and ethical conduct and for dealing } \\
\text { with allegations or evidence of misconduct. } \\
\text { - Prioritize the development of specialist training in ethical conduct for all staff working with } \\
\text { children and adolescents. } \\
\text { - Promote the establishment of a central resource and networking mechanism to provide } \\
\text { technical assistance to investigators (e.g., mechanisms for ongoing support during } \\
\text { implementation) following ethical standards. } \\
\text { - Visibly create an environment that promotes integrity, objectivity, and the highest ethical } \\
\text { standards, including design, implementation, and reporting. } \\
\text { Monitor and discuss adherence to ethical standards among all activities with children and } \\
\text { adolescents conducted by colleagues. }\end{array}$ \\
\hline $\begin{array}{l}\text { Community } \\
\text { groups and } \\
\text { advisory board } \\
\text { members }\end{array}$ & $\begin{array}{l}\text { - Provide local culturally specific information to investigators. } \\
\text { - Ensure that ethical standards are followed by monitoring activities. } \\
\text { - Inform community members about how children will be protected by the activity. }\end{array}$ \\
\hline $\begin{array}{l}\text { Ethical review } \\
\text { board members }\end{array}$ & $\begin{array}{l}\text { - Create and publicize criteria for ethical approval of new activities. } \\
\text { - Oversee ongoing activities to ensure they are conducted according to approved protocol, } \\
\text { that all participants are protected and that all information gathered is authentic and reliable. } \\
\text { - Make sure that all investigators receive written policies, procedures, and guidelines before } \\
\text { activities are initiated, and receive appropriate training in their implementation. }\end{array}$ \\
\hline $\begin{array}{l}\text { Program } \\
\text { managersl } \\
\text { researchers }\end{array}$ & $\begin{array}{l}\text { - Ensure compliance with requirements and recommendations of supervisory mechanisms } \\
\text { (e.g., ethical review and community advisory groups), and demonstrate to the review board } \\
\text { familiarity with the national and agency policies for working with children and adolescents. } \\
\text { - Include the views of community members by establishing a community advisory board. } \\
\text { - Promote the visible adoption of specific agency guidelines for the conduct of work involving } \\
\text { children and adolescents. If there is no policy for working with children, work to create one } \\
\text { (see the example Child Protection Policy and Procedures in Annex 4.2). } \\
\text { - Develop and implement training in ethical conduct for all staff who gather information among } \\
\text { children and adolescents. } \\
\text { - Make sure that local offices administering activities have appropriate budgetary resources to } \\
\text { meet the specific training needs of staff working with children and adolescents, such as } \\
\text { familiarity with policies and procedures for the protection of children, including their } \\
\text { psychosocial welfare. } \\
\text { Identify or develop a networking mechanism that can be used by all agencies working with } \\
\text { children and adolescents. Distribute and exchange information regarding implementation of } \\
\text { ethical standards. }\end{array}$ \\
\hline
\end{tabular}




\begin{tabular}{|c|c|}
\hline Data analysts & $\begin{array}{l}\text { - Assure adequate planning to support the practical value of the activity, the validity of the } \\
\text { expected results, and the ability to provide the protection promised (e.g., use "power" } \\
\text { calculations to make informed recommendations for study size to avoid using too many or } \\
\text { too few participants). } \\
\text { - Protect the privacy and confidentiality of participants. } \\
\text { - Design information-gathering tools (e.g., questionnaires, interview guides) that minimize } \\
\text { intrusion. } \\
\text { - Make sure that all data collection tools are locally reviewed and field-tested so that they } \\
\text { avoid potentially traumatic questions. }\end{array}$ \\
\hline $\begin{array}{l}\text { Supervisors of } \\
\text { data collectors } \\
\text { and program } \\
\text { staff }\end{array}$ & $\begin{array}{l}\text { - Train all staff who come into contact with children and adolescents and their information to } \\
\text { identify and follow ethical considerations when using any of the gathered information. } \\
\text { - Ensure that staff are familiar with procedures that protect children and adolescents from } \\
\text { abuse, and that they have a working knowledge of and access to local authority systems for } \\
\text { dealing with these issues. } \\
\text { - Supervise the conduct of interviewers and all investigators who come into contact with } \\
\text { children and adolescents. } \\
\text { - Raise questions relating to ethical concerns at regular meetings to sensitize staff and } \\
\text { provide a forum for discussion. } \\
\text { - Provide ongoing support and supervision to all staff who work with children and adolescents } \\
\text { (e.g., provide opportunities for peer discussions); identify an accessible individual who can } \\
\text { provide technical assistance when questions come up or when staff face new ethical } \\
\text { challenges during the activity. } \\
\text { - Introduce opportunities for community groups to help monitor whether or not ethical } \\
\text { procedures are being followed (e.g., hold community mobilization meetings, create local } \\
\text { advisory boards). }\end{array}$ \\
\hline Data collectors & $\begin{array}{l}\text { - Implement and uphold ethical standards in accordance with specific training. } \\
\text { - Stay in touch with individual community members and community groups to ensure cultural } \\
\text { acceptability of activities. }\end{array}$ \\
\hline $\begin{array}{l}\text { Administrative } \\
\text { staff }\end{array}$ & - Respect and uphold the confidentiality and anonymity of children's records. \\
\hline $\begin{array}{l}\text { Communications } \\
\text { staff }\end{array}$ & - Protect the identity of participants when communicating findings to audiences. \\
\hline
\end{tabular}


Part 3 - Summary and recommendations

62 Ethical Approaches to Gathering Information from Children and Adolescents in International Settings 


\section{Annexes}

\section{Annex 1: Child- and adolescent-focused resources}

1.1 A pproaches to working with children and adol escents

1.2 Child- and adolescent-related professional codes of conduct

\section{Annex 2: Legal and professional resources}

2.1 Historical devel opment of legal codes for medical research

2.2 Country-specific legal codes governing the conduct of research (limited list)

2.3 Professional codes of conduct

2.4 Key resources on ethical review boards

\section{Annex 3: Additional resources}

3.1 Ethics in social and behavioral research

3.2 Ethics in medical research involving children

3.3 Gender

\section{Annex 4: Sample documents}

4.1 Consent form

4.2 Child protection policy 


\section{Annex 1: Child- and adolescent-focused resources}

\subsection{A pproaches to working with children and adolescents}

Convention on the Rights of the Child.

http:/ / www.unicef.org/ crc/ crc.htm

Universal Declaration of Human Rights.

http:/ / www.un.org/ Overview/ rights.html

"Child needs assessment toolkit." Early Child Development Team of the World Bank.

http:/ / www.taskforce.org/ aidshome.html

Children in Focus: A M anual for Participatory Research with Children. Boyden, J. and J. Ennew. (eds.)

1997. Stockholm: Save the Children Sweden.

"Children on the brink 2004: A joint report of new orphan estimates and a framework for action." UNAIDS, UNICEF, and USAID. 2004. Washington, D.C.: USAID.

http:/ / www.unicef.org and http:/ / www.usaid.gov

"Children's participation in research: Reflections from the care and protection of separated children in emergencies project." Mann, G. and D. Tolfree. 2003. Stockholm: Save the Children Sweden.

http:/ / se-web-01.rb.se/ Shop/ Products/ Product.aspx?itemid=353

The Clinical Child Interview. Hughes, Jan and David Baker. 1990. New York: Guilford Press.

Conducting a Participatory Situation A nalysis of O rphans and V ulnerable Children A ffected by HIV /AIDS:

Guidelines and Tools. DeMarco, Renee. 2005. Arlington, VA, USA: Family Health International.

http:/ / www.fhi.org/ en/ HIVAIDS/ pub/ guide/ ovcguide.htm

A D eath in the Family: Orphans of the HIV Epidemic. Levine, C. (ed.). 1993. Relevant articles include "Mourning in secret: How youngsters experience a family death from AIDS" and "A dolescents in families with AIDS: Growing up with loss." N ew York: United Hospital Fund of New York. http:/ / www.uhfnyc.org/ pubs-stories3220/ pubs-stories_show.htm?doc_id=93805

Ethics, Social Research and Consulting with Children and Y oung People. Alderson, P. and V. Morrow. 2004. London: Barnardo's.

http:/ / www.barnardos.org.uk/ shop/ publications/ acatalog/ Publications_Shop_Society_124.html

"Framework for the protection, care and support of orphans and vulnerable children living in a world with HIV/ AIDS." UNICEF et al. 2004. New York: UNICEF.

http:/ / www.unicef.org/ aids/ Framework_English.pdf

H ow to Research the Physical and Emotional Punishment of Children. Ennew, J. and D.P. Plateau. 2004. Bangkok: Save the Children, Southeast, East Asia and Pacific Region.

Involving Y oung Researchers: H ow to Enable Y oung People to D esign and Conduct Research. Kirby, Perpetua. 1999. Joseph Rowntree Foundation.

"Listening to young voices: Facilitating participatory apprai sals on reproductive health with adolescents." Shah, M., R. Zambezi, and M. Simasiku. 1999. CARE International in Zambia and FOCUS on Young Adults.

http:/ / www.pathfind.org/ pf/ pubs/ focus/ RPPS-Papers/ pla1.pdf 
O rphans and $O$ ther Children M ade Vulnerable by HIV \& AIDS - P rinciples and O perational Guidelines for Programming. International Federation of Red Cross and Red Crescent Societies. 2002.

http:/ / www.ifrc.org/ what/ health/ tools/ orphans.asp

A Parrot on Your Shoulder: A Guide for People Starting to W ork with Orphans and V ulnerable Children. International HIV/ AIDS Alliance. 2004. Brighton, U.K.: International HIV/ AIDS Alliance. http:/ / www.aidsalliance.org/ sw7467.asp

PLA N otes (Participatory Learning and A ction). See in particular "Special issue on children's participation" (Issue 25, February 1996) and "Children's participation: Evaluating effectiveness" (Issue 42, October 2001). International Institute for Environment and Development. http:/ / www.iied.org/ sarl/ pla_notes/ backissues.html

"Psychosocial support," Building blocks: A frica-wide briefing notes-Resources for communities working with orphans and vulnerable children. International HIV/ AIDS Alliance. 2003. Brighton, U.K.:

International HIV/ AIDS Alliance. http:/ / www.aidsalliance.org/ sw9170.asp

"So you want to consult with children? A toolkit of good practice" and "So you want to involve children in research? A toolkit supporting children's meaningful and ethical participation in research relating to violence against children." Save the Children. 2004. Stockholm: Save the Children.

http:/ / www.savethechildren.net/ alliance/ resources/ publications.html.

Stepping Forward: Children and Y oung People's Participation in the D evelopment Process. Johnson, $\mathrm{V}$. et al. (eds.). 1998. Rugby, U.K.: Intermediate Technology Publications.

http:/ / www.developmentbookshop.com (use search tool).

Y oung People as Researchers: A Learning Resource Pack. Worrall, Steve. 2000. Save the Children UK and Joseph Rowntree Foundation.

\section{$1.2 \mathrm{Child}$ - and adolescent-related professional codes of conduct}

"Code of research ethics: a position paper of the Society for A dolescent Medicine." 2003. Journal of A dol escent $\mathrm{H}$ eal th 24:277-282.

http:/ / www.adolescenthealth.org/ researchethics.htm

"Guidelines for research on reproductive health involving adolescents." World Health

Organization.

http:/ / www.who.int/ reproductive-health/ hrp/ guidelines_adolescent.en.html

"Guidelines for research with children." National Children's Bureau.

http:/ / www.ncb.org.uk/ ourwork/ detail.asp?PID=144

National Association for the Education of Young Children

http:/ / www.naeyc.org/ about/ positions.asp

"Orphans and other vul nerable children support tool kit." 2005. International HIV/ AIDS Alliance and Family Heal th International.

http:/ / www.ovcsupport.net

Society for Research in Child Development

http:/ / www.srcd.org/ about.html \#standards 


\title{
Annex 2: Legal and professional resources
}

\subsection{H istorical development of legal codes for medical research}

"Belmont report: Ethical principles and guidelines for the protection of human subjects of research." National Commission for the Protection of Human Subjects of Research. 1978. U.S. Department of Health, Education, and Welfare, Office of the Secretary.

"International ethical guidelines for biomedical research involving human subjects." Council for International Organizations of Medical Sciences (CIOMS) in collaboration with the World Health Organization. Revised 2002. Geneva: CIOMS.

http:// www.cioms.ch/ frame_guidelines_nov_2002.htm.

"Trials of war criminals before the N uremberg military tribunals under Control Council Law No. 10." 2 (1949):181-182. Washington, D.C.: U.S. Government Printing Office. http:/ / ohsr.od.nih.gov/ guidelines/ nuremberg.html

"World Medical Association Declaration of Helsinki: Ethical principles for medical research involving human subjects." A dopted by the 18th WMA General Assembly, Helsinki, Finland, 1964. A mended by the 52nd WMA General Assembly, Edinburgh, Scotland, 2000.

http:/ / www.wma.net/ e/ policy/ b3.htm

\subsection{Country-specific legal codes governing the conduct of research (limited list)} International

"International ethical guidelines for biomedical research involving human subjects." Council for International Organizations of Medical Sciences (CIOMS). 1993.

\author{
Australia \\ "Guidelines on ethical matters in A boriginal and Torres Strait Islander health research." National \\ Health and Medical Research Council (NHMRC). 1991. \\ "N ational statement on ethical conduct in research involving humans." NHMRC. 1999. \\ http:/ / www7.health.gov.au/ nhmrc/ publications/ humans/ preamble.htm \\ Brazil \\ "Resolution N o. 196/ 96 on research involving human subjects." National Health Council. 1996. \\ English: http:/ / www.aids.gov.br/ resolution_196.htm \\ Portuguese: http:/ / conselho.saude.gov.br/ docs/ Resolucoes/ Reso196de96.doc \\ Addition: Resolution N o. 251. 1997. Addition: Resolution N o. 292. 1999.
}

\section{Canada}

"Tri-council policy statement: Ethical conduct for research involving humans, public works and government services." Medical Research Council of Canada, $\mathrm{N}$ atural Sciences and Engineering Research Council of Canada, and Social Sciences and Humanities Research Council of Canada. 1998.

\section{China}

"Committee on research involving human subjects, guidelines on ethical review of medical research." Ministry of Public Health. 1998. 


\section{Europe}

"Convention for the protection of human rights and dignity of the human being with regard to the application of biology and medicine: Convention on Human Rights and Biomedicine." Council of Europe. 1997. Oviedo: European Treaty Series.

http:/ / conventions.coe.int/ treaty/ en/ treaties/ html/ 164.htm

\section{Finland}

Medical Research Decree (986/ 1999).

The Patient Rights Act (785/ 1992).

\section{France}

"Law 88-1138: Regarding the protection of persons agreeing to biomedical research ('Huriet Law')."

\section{India}

"Ethical guidelines for social science research in health." N ational Committee for Ethics in Social Research in Health (NCESSRH). 2001.

"Ethical guidelines on biomedical research involving human subjects." Indian Council of Medical Research. 2000.

http:/ / www.icmr.nic.in/ ethical.pdf

\section{Nepal}

"Ethical guidelines for biomedical research involving human participants in N epal." Health

Research Council. 2000.

\section{Netherlands}

"Law of 26 February 1998, containing regulations with regard to medical-scientific research on humans." Staatblad (Official Law Gazette of the N etherlands) 161.

\section{New Zealand}

"Guidelines on ethics in health research." Health Research Council of New Zealand. 1997.

"Guidelines for researchers on health research involving Maori." Health Research Council of N ew Zealand. 1998.

\section{South Africa}

"Guidelines for good practice in the conduct of clinical trials in human participants in South Africa." Clinical Trials Working Group of the South A frican Department of Health. 2000. http:/ / 196.36.153.56/ doh/ docs/ policy/ trials/ trials-full.html

"Guidelines on ethics for medical research." South African Medical Research Council. Revised edition 1993.

http:/ / www.mrc.ac.za/ ethics/ ethics.htm

\section{Thailand}

"Rule of the Medical Council on the observance of medical ethics." Ministry of Public Health. 1995.

\section{Uganda}

"Guidelines for the conduct of health research involving human subjects in Uganda." National Consensus Conference on Bioethics and Health Research in Uganda. 1997.

\section{United Kingdom}

"Guidelines for good clinical practice in clinical trials." Medical Research Council (MRC). 1998. 
"Guidelines on the practice of ethics committees in medical research invol ving human subjects." Royal College of Physicians of London. 1996.

"Research involving human participants in developing societies: Ethical guidelines for MRCsponsored studies." MRC. 2004.

http:/ / www.mrc.ac.uk/ pdf-devsoc.pdf

"Responsibility in investigations on human participants and material and on personal information." MRC. 1992.

"UK good dinical practice in clinical trials." MRC. 1998.

http:/ / www.mrc.ac.uk/ pdf-ctg.pdf

\section{United States}

"Code of federal regulations: Title 45 public welfare, Part 46 protection of human subjects."

(Regulations for children are in Subpart D). U.S. Department of Health and Human Services. 2001. http:/ / www.hhs.gov/ ohrp/ humansubjects/ guidance/ 45cfr46.htm\#subpartd

\section{Zimbabwe}

"Guidelines for researchers and ethics review of committees in Zimbabwe." Medical Research

Council of Zimbabwe. 2004.

http:/ / www.afronets.org/ mrcz.php

\subsection{Professional codes of conduct}

African Evaluation Network

http:/ / www.afrea.org

American Anthropological Association http:/ / www.aaanet.org/ committees/ ethics/ ethcode.htm;

http:/ / www.aaanet.org/ committees/ ethics/ bp.htm

American Association for Marriage and Family Therapy

http:/ / www.aamft.org/ resources/ Irmplan/ ethics/ ethicscode2001.asp

American Association of Family and Consumer Sciences

http:/ / www.aafcs.org/ about/ ethics.html

American Counseling Association

http:/ / www.cacd.org/ codeofethics.html

American Political Science Association

http:/ / www.apsanet.org/ imgtest/ ethicsguideweb.pdf

American Psychological Association

http:/ / www.apa.org/ ethics/ code2002.html

American Sociological Association

http:/ / asanet.org/ galleries/ default-file/ Code\%20of\%20Ethics.pdf

American Statistical Association

http:/ / www.amstat.org/ profession/ index.cfm?fuseaction=ethicalstatistics

Association of Social Anthropologists

http:/ / www.theasa.org/ ethics.htm 
British Psychological Society http:/ / www.bps.org.uk/ documents/ Code.pdf

British Sociological Association

http:/ / www.britsoc.co.uk/ new_site/ user_doc/ Statement\%20of\%20Ethical\%20Practice.pdf

Canadian Code of Ethics for Psychologists

http:/ / www.cpa.ca/ ethics2000.html

Illinois Department of Children and Family Services (Standard ethics of child welfare policy)

http:/ / www.state.il.us/ DCFS/ docs/ CodeEthics.pdf

International Statistical Institute

http:/ / www.cbs.nl/ isi/ ethics.htm

Market Research Society

http:/ / www.mrs.org.uk/ standards/ children.htm

National Association of Social Workers.

http:/ / www.naswdc.org/ pubs/ code/ default.asp

National Council on Family Relations

http:/ / www.ncfr.org/ about_us/ g_governance_ethical.asp

Social Research Association

http:/ / www.the-sra.org.uk/ documents/ pdfs/ respect_code.pdf

\subsection{Key resources on ethical review boards}

"Federal policy for the protection of human subjects; notices and rules," Federal Register. 1991. 56:117. Also known as the "Common Rule," Subpart A requires the establishment of "institutional review boards" (IRBs). United States Government.

http:/ / www.hhs.gov/ ohrp/ references/ comrulp1.pdf

"Operational guidelines for ethics committees that review biomedical research." World Health Organization. 2000.

http:/ / www.who.int/ tdr/ publications/ publications/ ethics.htm

"Surveying and evaluating ethical review practices." World Health Organization. 2002.

http:/ / www.who.int/ tdr/ publications/ publications/ ethics2.htm 


\section{Annex 3: Additional resources}

\subsection{Ethics in social and behavioral research}

“Ethics and reproductive health." Family Health International. 2001. N etwork 21(2).

http:/ / www.fhi.org/ en/ RH/ Pubs/ Network/ v21_2/ index.html

Ethics Web

http:/ / www.ethicsweb.ca/ resources/ research/ index.html

"Experts weigh ethical issues in research with ethnic-minority youth." Carpenter, Siri. 2001. A PA

M onitor 32(9).

http:/ / www.apa.org/ monitor/ oct01/ weighissues.html

"Research ethics training curriculum." Family Health International.

http:/ / www.fhi.org/ en/ RH/ Training/ trainmat/ ethicscurr/ index.htm

"Research ethics and children." Fordham University Center for Ethics Education.

http:/ / www.fordhamethics.org/ links/ researchchildren.htm

"Bioethics resources on the Web." National Institutes of Health.

http:/ / www.nih.gov/ sigs/ bioethics/

"Human participant protections education for research teams." National Institutes of Health.

http:/ / cme.cancer.gov/ clinicaltrials/ learning/ humanparticipant-protections.asp

"Policy and guidelines on the inclusion of children as participants in research involving human subjects." National Institutes of Health. 1998.

grants.nih.gov/ grants/ guide/ notice-files/ not98-024.html

Plus questions and answers. 1999.

http:/ / grants.nih.gov/ grants/ funding/ children/ pol_children_qa.htm

"Program on ethical issues in international health research." Harvard School of Public Health. http:/ / www.hsph.harvard.edu/ bioethics/

Research for D evelopment: A Practical Guide. Laws, Sophie, Caroline Harper, and Rachel Marcus. 2002. London: Sage Publications.

"Web-based instruction on informed consent." University of Minnesota.

http:/ / www.research.umn.edu/ consent/ orientation.html

"Guide for interpreting the federal policy for the protection of human subjects." USAID.

http:/ / www.usaid.gov/ our_work/ global_health/ home/ TechA reas/ commrule.html

"Informed consent: From good intentions to sound practices." Wood, S. Y., B. A. Friedland, and C. E. McGrory. 2002. Population Council-E bert Program Publication Series. N ew York: Population Council.

http:/ / www.popcouncil.org/ pdfs/ ebert/ informedconsent.pdf

\subsection{Ethics in medical research involving children}

"Children as decision makers: Guidelines for pediatricians." King, N. M. P. and A. W. Cross. 1989. Journal of Pediatrics 115: 10-16.

Children as Research Subjects: Science, Ethics, and Law. Grodin, M. and L. Glantz (eds.). 1994. New York: Oxford University Press. 
The Ethical Conduct of Clinical Research Involving Children. Board on Health Sciences Policy (HSP) and Institute of Medicine (IOM). 2004. Washington, D.C.: The National A cademies Press. http:/ / books.nap.edu/ books/ 0309091810/ html/ index.html

"The ethics of research with children." Kodish, E. Online Ethics Center.

Retrieved March 12, 2004 from http:/ / onlineethics.org/ reseth/ mod/ childres.html

"Informed consent in the children's cancer group: Results of preliminary research." Kodish, E. et al. 1988. Cancer 82:2467-80.

Who Speaks for the Child? The Problems of Proxy Consent. Gaylin, W. and Macklin, R. 1982. New York: Plenum Press.

\subsection{G ender}

“DANIDA 's gender analysis training: A three module training package." Ministry of Foreign Affairs, Denmark. 1998.

“Good practice: Planning, conducting and evaluating 'tailored' gender training courses." DfID (UK Department for International Development). 1999.

http:/ / www.siyanda.org/ docs_gem/ index_implementation/ t_toolsmenu.htm

“H ow to integrate gender into HIV/ AIDS programs: Using lessons learned from USAID and partner organizations." USAID Interagency Gender Working Group. 2004.

http:/ / www.prb.org/ pdf04/ HowTolntegrGendrHIV.pdf 


\section{Annex 4: Sample documents}

\subsection{Consent form}

As indicated throughout this publication, all materials developed to support information gathering activities among young people should be devel oped in close consultation with local community members and stakeholders, in order to make sure that they are age appropriate and culturally acceptable. Especially when investigating potentially sensitive topics, consultation with a local specialist in psychosocial support is recommended. (See Guidelines E1-E6 for further information about consent procedures and materials.) Although all consent forms and information-gathering tools will be different according to their context, some example documents are included here in order to provide ideas.

Additional examples of consent forms and survey documents are available in the appendix of Conducting a Participatory Situation A nalysis of $\mathrm{O}$ rphans and $\mathrm{V}$ ulnerable Children A ffected by HIV /AIDS: Guidelines and Tools (FHI 2005).

\section{a. Example of a consent form developed to support a semi-structured questionnaire to be used with young people aged 10-14}

The following form was used only after the child's parent or guardian had agreed to their involvement. Interviewers were trained to use the words in capitals as cues for the essential elements to be discussed with the child.

BASELINE SURVEY: Questions for youth aged 10-14

INTRODUCTION: Hello! My name is .... I'm here for a study on behalf of XXXX, who are providing services here in [insert name of community] like [insert name of local services]

PURPOSE: We're talking with adults and children here to get information about their lives and how XXXX can provide them with support, especially in difficult times. The information we obtain will be used to assess how the services are going, and how XXXX can improve them.

IDENTITY AND INVOLVEMENT: I have already been talking to your parent/guardian about these things, and now we would like to try to understand what you think. So, I would like to know if you are [insert selected child's name]? Have you been interviewed in the past two weeks for this study?

Proceed only if identity is correct and no previous involvement.

FUNDING SOURCE: Consider if it's appropriate to mention: These services are being provided by XXXX, whose local partners are [insert name of local partners].

PROCEDURES INVOLVED: We would like to ask you some questions, in an interview which will take about 15-30 minutes. I would like to talk to you alone, but if you like, you can ask for a parent or guardian to be present at any time.

RISKS: Some of these questions might talk about things that some people find quite personal, or may be difficult to answer. If any of the questions make you feel uncomfortable or you don't want to answer them, you do not have to.

ABILITY TO SAY NO: Remember, you do not have to talk about anything you don't want to. This will not affect your ability to receive services now or in future. However, I would really appreciate it if you would answer the questions honestly and openly, so that we can find out what young people here in [insert name of community] really think. Your answers will be very important to us. We would really appreciate any help you 
can give us in finding out about children here. Do you have any questions about any of the things I have just said?

WHAT WILL HAPPEN WITH THE INFORMATION: We'll be asking children from different places all over the country the same questions. When the survey is finished, we will collect all the responses we have received here in [insert name of community] and keep them safe. Someone in the office will add them together. You will be able to find out about the results by contacting our partners here, like [name local partners, and make sure individual knows how to contact them if they want to].

CONFIDENTIALITY: If you agree to take part in this interview, the things you tell me will be confidential. That means they will be private between you and me. I am not going to write down your name. If you agree, you can indicate your agreement by making a mark here. Alternatively, I can sign here to say that I have witnessed your agreement.

SEEKING COMPREHENSION: Do you have any questions about any of the things I have just said? SEEKING VOLUNTARY AGREEMENT: Are you willing to participate in this interview? Do you want a parent or guardian to stay with us while we are talking?

In case of any queries contact:

Local Principal Investigator XXXX, Tel: XXXX or

Chairperson, Local Research Ethics Committee, Tel: XXXX

Youth/child agreement

$\square$ Adult present?

Witness

Date

(Specify who)

District Name

Area Name

Incomplete Interview Log—Child questionnaire

\begin{tabular}{|l|l|l|l|l|}
\hline & Visit 1 & Visit 2 & Visit 3 & Visit 4 \\
\hline Interviewer name & & & & \\
\hline Date of visit & & & & \\
\hline Time of visit & & & & \\
\hline $\begin{array}{l}\text { Next appointment: } \\
\text { date and time }\end{array}$ & & & & \\
\hline Comment code & & & & \\
\hline
\end{tabular}



Comment codes:
1 Appointment made for later today
2 Appointment made for another day
3 Refused to continue and no appointment made
4 Other (Specify)

\section{Interviewer}

Name

Signature

Date completed

Checked by supervisor

Name
Signature 


\section{b. Example of a consent form used with guardians}

\section{The following form was developed to support an exercise to develop a book collecting children's memories of their parents, and publishing them together with photographs.}

Source: "Telling our stories: Children deal with loss, grief and transition." CA RE International Zambia and FHI. 2003. http:/ / www.synergyaids.com/ caba/ documents/ telling\%20our\%20stories.pdf

\section{Children's Book Project \\ Care Zambia And Scope-OVC Publication of \\ Orphaned Children's Memories Of Parents With Photographs. \\ Lusaka, October 2002}

\section{Consent to take part in the Book Project}

SCOPE-OVC is developing a book where children between the ages of ten and fourteen, who have lost one or both parents, will write about their experiences and memories of their parents. We shall take photographs of the children to put in the book, along with their words. This book will be distributed widely in Zambia, throughout Africa and even overseas.

I am asking you to give us permission to include your child in a children's book for CARE Zambia and SCOPE-OVC. In order to make sure that you are informed about this book and your child's participation in the book, I will ask you to sign or make a mark in front of a witness that you have understood this form and agree to participate in the project. We will give you a copy of this form.

There may be words or concepts on this form that are unfamiliar to you. Please be sure to ask us to explain anything that may be unfamiliar to you.

This book will be important for the children who take part and it will help other children who have lost parents to remember their own parents when they read it. Children will realise that they are not alone in their thoughts and feelings.

This book will also be helpful to parents, guardians, teachers and all those who care for children who have lost parents. It will help all of us to gain a better understanding of issues for children who have lost their parents.

The book will also help teach other people about what is happening to your children and hopefully help raise support for issues related to orphans and vulnerable children in Zambia. SCOPE-OVC will use this book as an advocacy tool to help raise support for children in Zambia.

If you agree that your child will participate in the project, the following will happen.

1. In October and November 2002, the project leader will visit all parents/guardians to explain to you how your child/ward will take part in the book project. You can ask any questions in order to decide whether you want to give your child permission to take part.

All the children will have their photographs taken where they live and/or at school. These pictures will be used in the book.

You will sign another form to give permission for photographs to be taken and used in the book and for anything concerned with the book project.

2. In December 2002, all the children who agree to take part in the project will attend a five-day residential programme, which will be organised by SCOPE-OVC. With the help of counsellors and other project workers, children will be helped to talk or write about memories of their parents. This will be a chance for children to take part in other activities such as art, music and craftwork. Children will be looked after during this 
programme by trained counsellors. The stories written during this period will appear in the published book. There will be no financial cost to you if you participate in this project. All meals, accommodation and transportation will be provided by SCOPE-OVC.

We think it will be healthy and positive for children to participate in this project. They will be with other children who have lost their parents and will learn that they are not alone in their thoughts and feelings about having lost their parents. They will remember many good and happy times about their parents. They will have an opportunity to write and participate in other fun activities. Overall, it will be a positive and constructive activity for the children.

However, sometimes remembering what has been lost can cause pain and sorrow. It is possible that for a while after participating in the project, the child may feel sad or may exhibit different behaviours.

There are some places where you will be able to go to get some help if needed. We will provide you with a list of organisations in Lusaka that might be able to help you. Children will also be provided with this list when they are participating in the workshop.

Throughout this project, there will be experienced counsellors who can help the children to talk about difficult and upsetting topics. If the children are upset while they remember their parents or previous experiences, we will help them to deal with difficult and upsetting memories.

Since pictures will be included in the book with the stories, you should be aware that there is no confidentiality. Readers of the book will know who the children are and to whom the stories belong.

Guardians and children who take part in the book project will not be paid for participation but they will get some photographs and receive two copies of the book when it is published.

Participation in the project is completely voluntary. Your child does not have to participate in this project. Refusal to participate will not affect any benefit you may receive from SCOPE-OVC or any other project helping children or families.

You may also stop participating in this project at any time or refuse to allow your child's story to be published. There will be no retributions from deciding not to participate in the project in any capacity.

SCOPE-OVC will not profit from the book in any capacity. If the book is sold, all the money from the sale will be used by CARE Zambia and SCOPE-OVC for projects that help children.

If, at any time, you feel that you or your child have further questions or need more information during the course of this project, please contact the following persons:

Project Manager, Address, Telephone

Country Director, Address, Telephone 
Do you agree to allow your child to participate in this book project?

Signature of parent/guardian/custodian (Date)

Signature of Project Representative (Date)

Signature of Witness (Date)

\section{For the delivery of the book:}

PO Address:

Physical Address:

\section{Consent to use photographs:}

I, (Name)

Address

Being the parent/guardian/custodian of (Name of child):

Give permission to the photographers on the book project to take photographs of my child/ward.

I agree that any photographs taken of my child/ward, living or otherwise, for the CARE Zambia SCOPE-OVC children's book project can be used in the book and in any other printed or electronic form as long as this is part of the book project.

No photographs will be used for any other project without further permission being given by me.

No photographs will be sold for profit by the photographers or CARE Zambia and SCOPE-OVC.

Copyright for the photographs will remain with the photographers.

Signature of parent/guardian/custodian

(Date)

Signature of project representative

(Date)

Signature of photographer

(Date)

Signature of witness

(Date) 


\subsection{Child protection policy}

Excerpt from the International Save the Children Alliance's child protection policy, which provides a basic code of conduct for all investigators gathering information.

Source: "Save the Children: Child protection policy." International Save the Children Alliance. 2003. http:/ / www.savethechildren.net/ alliance/ resources/ publications.html.

\section{Child protection: code of conduct}

Staff and others must never:

- hit or otherwise physically assault or physically abuse children

- develop physical/ sexual relationships with children

- develop relationships with children which could in any way be deemed exploitative or abusive

- act in ways that may be abusive or may place a child at risk of abuse

- use language, make suggestions or offer advice which is inappropriate, offensive or abusive

- behave physically in a manner which is inappropriate or sexually provocative

- have a child/ children with whom they are working to stay overnight at their home unsupervised

- sleep in the same room or bed as a child with whom they are working

- do things for children of a personal nature that they can do for themselves

- condone, or participate in, behaviour of children which is illegal, unsafe or abusive

- act in ways intended to shame, humiliate, belittle or degrade children, or otherwise perpetrate any form of emotional abuse

- discriminate against, show differential treatment, or favour particular children to the exclusion of others.

This is not an exhaustive or exclusive list. The principle is that staff should avoid actions or behaviour which may be construed as poor practice or potentially abusive.

It is important for all staff and others in contact with children to:

- be aware of situations which may present risks and manage these

- plan and organise the work and the workplace so as to minimise risks

- as far as possible, be visible in working with children

- ensure that a culture of openness exists to enable any issues or concerns to be raised and discussed

- ensure that a sense of accountability exists between staff so that poor practice or potentially abusive behaviour does not go unchallenged

- talk to children about their contact with staff or others and encourage them to raise any concerns

- empower children - discuss with them their rights, what is acceptable and unacceptable, and what they can do if there is a problem.

In general it is inappropriate to:

- spend excessive time alone with children away from others

- take children to your home, especially where they will be alone with you. 

Program managers and researchers often gather information from children and adolescents in order to develop and evaluate appropriate responses to their needs. During information gathering, children and adolescents require protection and respect in accordance with the highest ethical standards. Strict adherence to ethical standards is critical for information gathering in all cases, but extra precautions must be in place for young people, since they are especially vulnerable to exploitation, abuse, or other unintended harmful outcomes of the information gathering process. Working ethically with children and adolescents to recognize and respond to their needs also requires that program managers and researchers seek out and include the views of children and adolescents, as part of a broadly participatory approach.

In response to a growing need, this publication was developed to provide practical recommendations on how to gather data among young people in a way that minimizes harm and maximizes benefits, based on an international multi-disciplinary expert consultative process.

Horizons is a global operations research program designed to identify and test strategies to improve HIV/AIDS prevention, treatment, and care programs and services. Horizons is implemented by the Population Council in collaboration with the International Center for Research on Women (ICRW), International HIV/AIDS Alliance, Program for Appropriate Technology in Health (PATH), Tulane University, Family Health International (FHI), and Johns Hopkins University. Horizons is funded by the President's Emergency Plan for AIDS Relief through the U.S. Agency for International Development (USAID).

For more information, please contact: Horizons/Communications Unit 4301 Connecticut Ave NW

Suite 280

Washington, DC 20008

horizons@pcdc.org

www.popcouncil.org/horizons

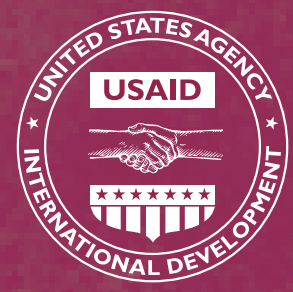

